\title{
OPTIMAL MULTIPLE STOPPING AND VALUATION OF SWING OPTIONS
}

\author{
RENÉ CARMONA AND NIZAR TOUZI
}

\begin{abstract}
The connection between optimal stopping of random systems and the theory of the Snell envelope is well understood, and its application to the pricing of American contingent claims is well known. Motivated by the pricing of swing contracts (whose recall components can be viewed as contingent claims with multiple exercises of American type) we investigate the mathematical generalization of these results to the case of possible multiple stopping. We prove existence of the multiple exercise policies in a fairly general set-up. We then concentrate on the Black-Scholes model for which we give a constructive solution in the perpetual case, and an approximation procedure in the finite horizon case. The last two sections of the paper are devoted to numerical results. We illustrate the theoretical results of the perpetual case, and in the finite horizon case, we introduce numerical approximation algorithms based on ideas of the Malliavin calculus.
\end{abstract}

\section{INTRODUCTION}

The motivation for the present study comes from the commodity markets, and especially the energy markets where the lack of standardization and the complexity of many contracts has attracted our attention. Commodity contracts can be extremely involved and many energy structured products are truffled with embedded options which are too often neither identified nor priced appropriately.

In this paper we concentrate on the mathematical analysis of options with multiple exercises of the American type. When embedded in base delivery contracts, these options are sometimes called swing options. In a vibrant industry sector with a multitude of tailor made contracts negotiated over the counter, the term swing has been used for many different things, and it is important to specify what we do have in mind when we use this terminology. The types of contracts containing the swing options we are considering are described in detail in [2] and [16], and they are slightly different from the gas sale agreements (GSA) discussed in [10]. These contracts for the covenants are not the same for the buyer and the seller. The continuous time finance problems considered in this paper, are addressing the pricing and optimal exercises challenges of the recall options contained in the buyer side of the swing contracts. To keep things at a reasonable level of complexity, we assume the purpose of this paper, that the holder of a swing option is given the opportunity to exercise several rights, and that she has total freedom in the choice of the timing of these exercises. This outstanding feature of the embedded options is reminiscent of American contingent claims with multiple exercises, and despite the existence of several numerical pricing algorithms such as [24], [16], [10], or [2] to name a few, it seems that a rigorous mathematical analysis of the valuation of this specific form of multiple optionality has not been provided in the existing literature. The purpose of this paper is to fill this gap by offering a first mathematical analysis of these options with multiple American exercises.

Date: October 29, 2004.

The authors are grateful for many interesting discussions with Bruno Bouchard. 
The optimal stopping problem is a "classic" whose solution had far reaching applications in many different fields. In its most general form, its mathematical solution is provided by the elegant theory of the so-called Snell envelope. See for example [23] for a clear presentation in the discrete case, and [12] for a thorough discussion in the context of optimal control of continuous time stochastic processes. The reader mostly interested in the application to the pricing of contingent claims with American exercise is referred to the appendices A and D of [17] for a self contained exposé of the continuous time theory. Practical applications requiring a generalization of the optimal stopping problem to possibly multiple stops are plentiful. A version of such a generalization in the discrete time case goes back to [14] and can be found in the book of Cairoli and Dalang [6].

After a short review of the standard results from optimal stopping which are used in the analysis of single American exercises, we introduce the inductive hierarchy of Snell envelopes needed in the multiple exercise case, and we give results on the existence and the characterization of a set of optimal stopping/exercise times under an assumption which guarantees that all these Snell envelopes have continuous paths. These results are straightforward generalizations of the classical theory. They are included for the sake of completeness as a way to introduce the notation. We deliberately excluded the case where the Snell envelope may present a discontinuity in order to avoid technical difficulties which are beyond the scope of this paper.

We then concentrate on the geometric Brownian motion framework of the Black-Scholes theory, and we identify explicitly the solution in the case of perpetual swings generalizing the classical problem of the exercise of a perpetual American put option. In this case the optimal exercise regions form a strictly decreasing sequence of intervals and the exercise times are given by the hitting times of the ordered set of their boundaries which we identify. See also [7] for the case of one dimensional diffusions.

The final section of this paper applies the Monte Carlo approximation method suggested in Lions and Regnier [19] and further developed by Bouchard and Touzi [5] to our context of multiple stopping. The above papers make an extensive use of the Malliavin calculus. This is obviously an overkill in the situation considered in this paper. Indeed, the price process is log-normal in the Black-Scholes framework, and explicit computations are possible. We review this approach while streamlining its dependence upon the sophisticated tools of the Malliavin calculus, and by providing a direct and self-contained account of the various steps.

After this paper was completed, we learned of the existence of [22] where the authors derive Monte Carlo upper and lower bounds by extending Rogers' duality strategy to American options with multiple exercises. Motivated by the pricing of chooser flexible caps, they study what could be viewed as the buyer side of a swing option.

\section{Optimal Stopping And American Options}

Let $(\Omega, \mathcal{F}, \mathbb{P})$ be a complete probability space, and $\mathbb{F}=\left\{\mathcal{F}_{t}\right\}_{t>0}$ be a filtration satisfying the usual assumptions, i.e. an increasing right continuous family of sub- $\sigma$-algebras of $\mathcal{F}$ such that $\mathcal{F}_{0}$ contains all the $\mathbb{P}$-null sets. We also assume that $\mathcal{F}_{0}$ contains only sets of probability zero or one, and we denote by $\mathcal{S}$ the set of all the $\mathbb{F}$-stopping times. Let $X=\left\{X_{t}\right\}_{t \geq 0}$ be a non-negative $\mathbb{F}$-adapted process satisfying the following properties:

$$
\text { the process } X \text { is continuous a.s }
$$


and

$$
\mathbb{E}\{\bar{X}\}<\infty \quad \text { where } \bar{X} \text { is defined by } \bar{X}=\sup _{t \geq 0} X_{t} .
$$

Each random variable $X_{t}$ has the interpretation of a reward or payoff if we stop the process or if we use one exercise right at time $t$. We also fix a time $T \in(0, \infty]$ which has the interpretation of maturity, i.e. the time of expiration of our right to stop the process or exercise. In order to include both the cases of finite and infinite horizon stopping problems in the same framework, we assume that:

$$
X . \equiv 0 \quad \text { on }(T, \infty) .
$$

In the infinite horizon case $T=+\infty$, we set

$$
\mathcal{F}_{\infty}=\sigma\left\{\cup_{0 \leq t<\infty} \mathcal{F}_{t}\right\} \quad \text { and } \quad X_{\infty}=\limsup _{t \rightarrow \infty} X_{t}
$$

We denote by $\mathcal{S}_{T}$ the collection of all the $\mathbb{F}$-stopping times with values in $[0, T]$. Given a stopping time $\theta \in \mathcal{S}_{T}$, we denote by $\mathcal{S}_{\theta, T}$ the subset of $\mathcal{S}_{T}$ consisting of all the stopping times $\tau \geq \theta$ a.s.

We now review the notation and the results of the classical treatment of the optimal stopping problem which we will need in our analysis of the optimal multiple stopping problem associated to the valuation of swing options. The interested reader can find a more general treatment in [12] and [17]. In particular, the presentation of [12] avoids the continuity condition (1) used here. In any case, the classical optimal stopping problem consists in the computation of the supremum

$$
\hat{X}_{0}=\sup _{\tau \in \mathcal{S}_{T}} \mathbb{E}\left\{X_{\tau}\right\} .
$$

together with the possible characterization of the stopping times at which the supremum is attained.

2.1. Snell Envelope and Optimal Stopping Times. The solution of the optimal stopping problem (4) is best understood by introducing the family $\left\{\hat{X}_{\theta} ; \theta \in \mathcal{S}_{T}\right\}$ of random variables defined by:

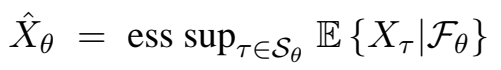

These random variables satisfies the dynamic programming principle:

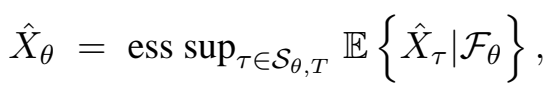

for all $\theta \in \mathcal{S}_{T}$. The $\mathbb{F}$-adapted process $\hat{X}=\left\{\hat{X}_{t}\right\}_{t>0}$ is a super-martingale, which is continuous in expectation, i.e. $\mathbb{E}\left\{X_{\tau_{n}}\right\} \longrightarrow \mathbb{E}\left\{X_{\tau}\right\}$ for all sequences $\left(\tau_{n}\right)_{n \geq 0} \subset \mathcal{S}$ with $\tau_{n} \rightarrow \tau$ a.s because of (1) and (2), and hence has a càdlàg modification of the process $\hat{X}$ which we still denote $\hat{X}$. It is called the Snell envelope of the process $X$. It can be characterized as the smallest super-martingale which dominates $X$. Moreover, each random variable $\hat{X}_{\theta}$ coincides with the evaluation of the process $\hat{X}$ at the stopping time $\theta$, i.e.

$$
\hat{X}_{\theta}(\omega)=\hat{X}_{\theta(\omega)}(\omega)
$$

We next define the stopping time

$$
\theta_{0}^{*}=\inf \left\{t \geq 0 ; \hat{X}_{t}=X_{t}\right\} .
$$


By right continuity of the sample paths we have $\hat{X}_{T}=X_{T}$, so $\theta_{0}^{*} \leq T$ a.s., and using the properties (1)-(2) of the process $X$, it can be proved that the stopped process $\hat{X}^{\theta_{0}^{*}}=\left\{\hat{X}_{t \wedge \theta_{0}^{*}}\right\}_{t \geq 0}$ is a martingale. As a consequence we see that

$$
\hat{X}_{0}=\mathbb{E}\left\{\hat{X}_{\theta_{0}^{*}}\right\}=\mathbb{E}\left\{X_{\theta_{0}^{*}}\right\}
$$

which shows that $\theta_{0}^{*}$ is an optimal stopping time for the problem (4). More generally, for any $\tau \in \mathcal{S}_{T}$, the random variable

$$
\theta_{\tau}^{*}=\inf \left\{t \geq \tau ; \hat{X}_{t}=X_{t}\right\}
$$

is less than or equal to $T$ a.s. because $\hat{X}_{T}=X_{T}$ when $T<\infty$. So it defines a stopping time in $\mathcal{S}_{\tau, T}$ and, by continuity of $X$, it follows that the stopped process $\left\{\hat{X}_{t \wedge \theta_{\tau}^{*}}\right\}_{t \geq \tau}$ is a martingale. Therefore:

$$
\hat{X}_{\tau}=\mathbb{E}\left\{\hat{X}_{\theta_{\tau}^{*}} \mid \mathcal{F}_{\tau}\right\}=\mathbb{E}\left\{X_{\theta_{\tau}^{*}} \mid \mathcal{F}_{\tau}\right\},
$$

and $\theta_{\tau}^{*}$ is an optimal stopping time for the problem (5) in the sense that the essential supremum appearing in (5) is attained for $\tau=\theta_{\tau}^{*}$.

2.2. Doob-Meyer Decomposition and Maximal $L^{p}$-Inequality. Since the Snell envelope $\hat{X}$ of the process $X$ is a càdlàg supermartingale, it follows from the Doob-Meyer decomposition theorem together with condition (2) that it admits the representation

$$
\hat{X}_{t}=M_{t}-A_{t} \text { for } 0 \leq t \leq T,
$$

where $M$ is a uniformly integrable càdlàg martingale, and $A$ is a non-decreasing $\mathbb{F}$-adapted process with $A_{0}=0$, and $\mathbb{E}\left\{A_{T}\right\}<\infty$. Since $X$ is continuous, we also have that $A$ is continuous and

$$
\int_{0}^{T} \mathbf{1}_{\left\{\hat{X}_{t}>X_{t}\right\}} d A_{t}=0
$$

See Karatzas and Shreve [17], Theorem D13. A consequence of this result is that a sample path of the Snell envelope $\hat{X}$ is continuous if and only if the corresponding sample path of the martingale part $M$ is continuous. This remark implies that the sample paths of the Snell envelope are almost surely continuous when the filtration $\mathbb{F}$ is generated by a Brownian motion. Indeed, in this case every martingale has continuous sample paths since it can be represented as a stochastic integral with respect to the Brownian motion. We shall make use of this result later in the paper.

Whenever the reward process $X$ satisfies the integrability condition:

$$
\mathbb{E}\left\{\bar{X}^{p}\right\}<\infty \text { for some } p>1
$$

(which is stronger than condition (2)) we can define the uniform integrable martingale $\bar{X}=\left\{\bar{X}_{t}\right\}_{0 \leq t \leq T}$ by $\bar{X}_{t}=\mathbb{E}\left\{\bar{X} \mid \mathcal{F}_{t}\right\}$ for $0 \leq t \leq T$, and use Doob's maximal inequality in its $L^{p}$ form

$$
\mathbb{E}\left\{\sup _{0 \leq t \leq T} \hat{X}_{t}^{p}\right\} \leq \mathbb{E}\left\{\sup _{0 \leq t \leq T} \bar{X}_{t}^{p}\right\} \leq\left(\frac{p}{p-1}\right)^{p} \mathbb{E}\left\{\bar{X}^{p}\right\}<\infty .
$$

Hence, the Snell envelope $\hat{X}$ inherits the $L^{p}$-integrability of the maximum from the underlying process $X$. As a consequence we see that

$$
\text { the process } \hat{X} \text { is left-continuous in expectation. }
$$


Indeed, if $\left\{\tau_{n}\right\}_{n \geq 0}$ is an increasing sequence of stopping times with $\tau_{n} \nearrow \tau \in \mathcal{S}$, then, $\hat{X}_{\tau_{n}} \longrightarrow \hat{X}_{\tau}$ a.s. by continuity of $\hat{X}$. Now, observe that

$$
\mathbb{E}\left\{\hat{X}_{\tau_{n}}^{p}\right\} \leq \mathbb{E}\left\{\sup _{0 \leq t \leq T} \hat{X}_{t}^{p}\right\}<\infty,
$$

so that the sequence $\left\{\hat{X}_{\tau_{n}}\right\}_{n \geq 0}$ is uniformly integrable, and (9) follows.

2.3. Optimal Multiple Stopping. From now on, we assume that the filtration $\mathbb{F}$ satisfies the additional requirements:

$$
\left\{\begin{array}{c}
\text { The filtration } \mathbb{F} \text { is left continuous and } \\
\text { every } \mathbb{F} \text {-adapted martingale has continuous sample paths. }
\end{array}\right.
$$

This condition guarantees that the Snell envelop of a continuous process is continuous. This property will be needed in the subsequent analysis which involves a composition of Snell envelopes. The case of discontinuous processes leads to technical problems which are beyond the scope of this paper.

Let $X$ be a non-negative $\mathbb{F}$-adapted process satisfying conditions (1), (2) and (8). Recall that the finite maturity framework is captured by our convention (3). We let $\overline{\mathcal{S}}_{T}$ be the set of all $\mathbb{F}$-stopping times valued in $[0, T] \cup\{T+\}$, and set

$$
X_{T+} \equiv 0 .
$$

Remark 1. In the case $\mathbb{F}=\mathbb{F}^{X}$ of the completion of the canonical filtration generated by a process $X, \mathbb{F}^{X}$ is left-continuous whenever the process $X$ has left-continuous sample paths.

We fix an integer $\ell \geq 1$ and a positive constant $\delta$. Here, $\ell$ represents the number of recall rights we can exercise, while $\delta$ is the length of the refracting time interval which needs to separate two successive exercises. This assumption on the separation of the exercise times prevents them from bunching up together on top of the optimal stopping time for the classical case reviewed in the previous section. But it is important to emphasize that we do not make this assumption for mathematical convenience. As reported in [16] the existence of such a refracting time period is part of the actual swing contracts traded in the energy markets. We shall denote by $\overline{\mathcal{S}}_{T}^{(\ell)}$ the collection of all vectors of stopping times $\vec{\tau}=\left(\tau_{1}, \ldots, \tau_{p}\right)$ such that

$$
\tau_{1} \leq T \text { a.s. and } \tau_{i}-\tau_{i-1} \geq \delta \text { on }\left\{\tau_{i-1} \leq T\right\} \text { a.s. for all } i=2, \ldots, \ell .
$$

Motivated by the valuation of swing options with multiple American exercises, we define the optimal multiple stopping problem by:

$$
Z_{0}=\sup _{\vec{\tau} \in \overline{\mathcal{S}}^{(\ell)}} \mathbb{E}\left\{X_{\vec{\tau}}\right\} \quad \text { where } \quad X_{\vec{\tau}}=\sum_{i=1}^{\ell} X_{\tau_{i}} .
$$

In the above multiple stopping problem, the holder of the option is allowed to exercise her rights to the reward/payoff given by the process $X$ at $\ell$ different times of her choosing. Notice that the holder of the option can decide not to use all her exercise rights by setting the last stopping times to $T+$. Such strategies could be desirable in this context because of the presence of the refracting period $\delta$, which may lead the investor to sacrifice an exercise right in order to benefit from a potential better future exercise. 
In order to characterize this multiple optimal stopping problem, we introduce the sequence of Snell envelopes :

$$
Y^{(0)} \equiv 0 \quad \text { and } \quad Y^{(i)}=\widehat{X^{(i)}} \text { for } i=1, \ldots, \ell,
$$

where, for each integer $i=1, \ldots, \ell$, the $i$-exercise reward process $X^{(i)}$ is defined by:

$$
X_{t}^{(i)}=X_{t}+\mathbb{E}\left\{Y_{t+\delta}^{(i-1)} \mid \mathcal{F}_{t}\right\}, \quad \text { for } 0 \leq t \leq T-\delta,
$$

and, whenever $T<\infty$,

$$
X_{t}^{(i)}=\hat{X}_{t}, \text { for } T-\delta<t \leq T .
$$

In order to apply the results of the single optimal stopping theory in this context, we need to ensure that the iterated reward processes $X^{(i)}$ inherit conditions (1), (2) and (8).

Lemma 1. Let us assume that condition (10) holds and that the process $X$ is continuous and satisfies condition (8). Then, for all $i=1, \cdots, \ell$, the process $X^{(i)}$ is continuous in expectation, and satisfies

$$
\mathbb{E}\left\{\bar{X}^{(i)^{p}}\right\}<\infty \text { where } \bar{X}^{(i)}=\sup _{0 \leq t \leq T} X_{t}^{(i)}
$$

Proof. Since $X^{(1)}=X$, the statement of the lemma holds trivially for $i=1$. Using the properties of the Snell envelope recalled in Subsection 2.2, and the properties of the filtration, we see that the processes $X^{(i)}$ and $\hat{X}^{(i)}$ inherit the pathwise continuity from $X$ and $\hat{X}^{(i-1)}$ for every $i \geq 1$.

We now prove that $\mathbb{E}\left\{\bar{X}^{(i)^{p}}\right\}<\infty$. We proceed by induction. For $i=1$, the result is just a restatement of condition (8). So we assume that $\mathbb{E}\left\{\bar{X}^{(i-1)^{p}}\right\}<\infty$, and we prove that $\mathbb{E}\left\{\bar{X}^{(i)^{p}}\right\}<\infty$. The martingale inequality implies that:

$$
\mathbb{E}\left\{\sup _{0 \leq t \leq T} Y_{t}^{(i-1)^{p}}\right\} \leq \mathbb{E}\left\{\sup _{0 \leq t \leq T} \mathbb{E}\left\{\bar{X}^{(i-1)^{p}} \mid \mathcal{F}_{t}\right\}^{p}\right\} \leq\left(\frac{p}{p-1}\right) \mathbb{E}\left\{\bar{X}^{(i-1)^{p}}\right\}<\infty
$$

from which we conclude that

$$
\mathbb{E}\left\{\bar{X}^{(i) p}\right\}^{1 / p} \leq \mathbb{E}\left\{\bar{X}^{p}\right\}^{1 / p}+\mathbb{E}\left\{\sup _{0 \leq t \leq T} Y_{t}^{(i-1) p}\right\}^{1 / p}<\infty .
$$

Next we identify a set of optimal stopping times for the multiple stopping problem. Let us set:

$$
\tau_{1}^{*}=\inf \left\{t \geq 0 ; Y_{t}^{(\ell)}=X_{t}^{(\ell)}\right\}
$$

Observe that $\tau_{1}^{*} \leq T$. Next for $2 \leq i \leq \ell$, we define

$$
\tau_{i}^{*}=\inf \left\{t \geq \delta+\tau_{i-1}^{*} ; Y_{t}^{(\ell-i+1)}=X_{t}^{(\ell-i+1)}\right\} \mathbf{1}_{\left\{\delta+\tau_{i-1}^{*}<T\right\}}+(T+) \mathbf{1}_{\left\{\delta+\tau_{i-1}^{*} \geq T\right\}} .
$$

Clearly, $\overrightarrow{\tau^{*}}=\left(\tau_{1}^{*}, \ldots, \tau_{p}^{*}\right) \in \mathcal{S}_{T}^{(\ell)}$. We are now ready to prove the main result of this section.

Theorem 1. If we assume that condition (10) holds and that the process $X$ satisfies (1), (2) and (8), then

$$
Z_{0}=Y_{0}^{(p)}=\mathbb{E}\left\{X_{\vec{\tau}^{*}}\right\} .
$$


Proof. Let $\vec{\tau}=\left(\tau_{1}, \ldots, \tau_{p}\right)$ be an arbitrary element in $\mathcal{S}_{T}^{(\ell)}$. For ease of notation, we set $\bar{\tau}_{i}:=\tau_{\ell-i+1}$. Let us set $X^{(0)} \equiv 0$. A straightforward inductive argument can be used to prove

$$
\mathbb{E}\left\{X_{\vec{\tau}}\right\} \leq \mathbb{E}\left\{X_{\bar{\tau}_{i}}^{(i)}+\mathbf{1}_{\{i<\ell\}} \sum_{j=i+1}^{\ell} X_{\bar{\tau}_{j}}\right\}
$$

for all $0 \leq i \leq \ell$. Now, using (15), together with the classical optimal stopping theorem, we see that:

$$
\mathbb{E}\left\{X_{\vec{\tau}}\right\} \leq \mathbb{E}\left\{X_{\tau_{1}}^{(p)}\right\} \leq Y_{0}^{(p)}=\mathbb{E}\left\{X_{\tau_{1}^{*}}^{(p)}\right\}=\mathbb{E}\left\{X_{\tau_{1}^{*}}+\mathbb{E}\left\{Y_{\delta+\tau_{1}^{*}}^{(p-1)} \mid \mathcal{F}_{\tau_{1}^{*}}\right\}\right\} .
$$

Next, we notice that the stopped sequence $\left\{Y_{t \wedge \tau_{2}^{*}}^{(p-1)} ; t>\delta+\tau_{1}^{*}\right\}$ is a martingale. We then deduce from the previous inequality that:

$$
\begin{aligned}
\mathbb{E}\left\{X_{\vec{\tau}}\right\} \leq Y_{0}^{(p)} & \leq \mathbb{E}\left\{X_{\tau_{1}^{*}}+\mathbb{E}\left\{Y_{\tau_{2}^{*}}^{(p-1)} \mid \mathcal{F}_{\tau_{1}^{*}}\right\}\right\} \\
& =\mathbb{E}\left\{X_{\tau_{1}^{*}}\right\}+\mathbb{E}\left\{Y_{\tau_{2}^{*}}^{(p-1)}\right\} .
\end{aligned}
$$

By repeatedly using the above argument, we see that

$$
\mathbb{E}\left\{X_{\vec{\tau}}\right\} \leq Y_{0}^{(p)} \leq \mathbb{E}\left\{X_{\tau_{1}^{*}}+\ldots+X_{\tau_{2}^{*}}\right\},
$$

proving the optimality of the vector of stopping times $\left(\tau_{1}^{*}, \ldots, \tau_{p}^{*}\right)$ for the problem $Z_{0}$, together with the equality $Z_{0}=Y_{0}^{(p)}$.

We next introduce the stopping times

$$
\zeta_{i}^{*}=\inf \left\{t \geq \delta+\tau_{i-1}^{*} ; Y_{t}^{(i)}=X_{t}^{(i)}\right\} \mathbf{1}_{\left\{\tau_{i-1}^{*}<T\right\}}+(T+) \mathbf{1}_{\left\{\tau_{i-1}^{*} \geq T\right\}} .
$$

Using the definition of $\theta_{\tau}^{*}$ given in (7), we see that $\zeta_{i}^{*}=\theta_{\delta+\tau_{i-1}^{*}}^{*}$ on $\left\{\tau_{i-1}^{*}<T\right\}$. In fact, the stopping time $\zeta_{i}^{*}$ is the first optimal stopping time for the single exercise/stopping problem $(p=1)$ starting from time $\delta+\tau_{i+1}^{*}$, where $\tau_{i+1}^{*}$ is an optimal stopping time for the exercise of the $(i+1)$-th right. Our next result confirms the intuitive belief that one should exercise earlier if one has more than one right.

Proposition 1. For any integer $i=1, \ldots, p$, we have :

$$
Y_{t}^{(i)} \leq Y_{t}^{(1)}+\mathbb{E}\left\{Y_{t+\delta}^{(i-1)} \mid \mathcal{F}_{t}\right\}, \quad t \geq 0, \text { and } \tau_{i}^{*} \leq \zeta_{i}^{*} .
$$

Proof. Let us set $V_{t}^{(i)}=Y_{t}^{(1)}+\mathbb{E}\left\{Y_{t+\delta}^{(i-1)} \mid \mathcal{F}_{t}\right\}$. From the supermartingale property of the processes $Y^{(1)}$ and $Y^{(i-1)}$, together with the inequality $Y^{(1)} \geq X$, we see that $V^{(i)}$ is a supermartingale which dominates $X^{(i)}$. We then conclude that $Y^{(i)} \leq V^{(i)}$, whence

$$
X_{t}^{(i)}=X_{t}+\mathbb{E}\left\{Y_{t+\delta}^{(i-1)} \mid \mathcal{F}_{t}\right\} \leq Y_{t}^{(i)} \leq Y_{t}^{(1)}+\mathbb{E}\left\{Y_{t+\delta}^{(i-1)} \mid \mathcal{F}_{t}\right\}
$$

We get $X_{\zeta_{i}^{*}}^{(i)}=Y_{\zeta_{i}^{*}}^{(i)}$ as an easy consequence of this inequality, and therefore we can conclude that $\tau_{i}^{*}$ $\leq \zeta_{i}^{*}$ by definition of $\tau_{i}^{*}$. 
We conclude this section by examining the case where the reward process $X$ is a submartingale. It is well-known that this implies that the maturity $T$ is an optimal stopping time for the (single) optimal stopping problem. This applies for instance to call options, and provides the equivalence between European and American call options, when the underlying asset does not deliver any dividends.

Proposition 2. Assume that $X$ is a submartingale, and let $T<\infty$ be a finite maturity date. Then

$$
\hat{X}_{t}^{\ell}=\sum_{i=1}^{\ell} \mathbb{E}\left\{X_{T-i \delta}\right\} \mathbf{1}_{t \leq T-i \delta}
$$

Proof. It is sufficient to notice that for $i=1, \ldots, \ell$ and $t \leq T-i \delta$, we have $X_{t}^{(i)}=X_{t}+$ $\mathbb{E}\left\{\hat{X}_{t+\delta}^{(i-1)} \mid \mathcal{F}_{t}\right\}$ inherits the submartingale property of $X$.

\section{Perpetual Swing Options in the Black-Scholes Framework}

In the previous section, we proved the existence of an optimal vector of stopping times, and we outlined an algorithm to construct such a vector by considering a cascade of Snell envelopes. There is no hope to get a more explicit characterization in such a generality. In this section, we concentrate on the problem of the optimal exercise of perpetual (i.e. $T=+\infty$ ) swing options in the Black-Scholes framework, and we identify the optimal exercise times as hitting times of a set of thresholds for which we provide a constructive algorithm.

3.1. The Black-Scholes Set-up. Let $W$ be an $\mathbb{R}$-valued Brownian motion on the probability space $(\Omega, \mathcal{F}, \mathbb{P})$, and denote by $\mathbb{F}=\left\{\mathcal{F}_{t}\right\}_{t \geq 0}$ the associated completed filtration. Notice that $\mathbb{F}$ satisfies condition (10). Let us fix a reward/payoff function $\phi: \mathbb{R}_{+} \longrightarrow \mathbb{R}_{+}$and let us consider a reward/payoff process of the form $\phi\left(X_{t}\right)$ where the process $X=\left\{X_{t}\right\}_{t}$ is now defined by:

$$
X_{t}=X_{0} \exp \left[\left(r-\frac{\sigma^{2}}{2}\right) t+\sigma W_{t}\right], \quad t \geq 0
$$

where $r$ and $\sigma$ are two positive parameters standing for the short interest rate and the volatility. Notice that we changed the notation and that the process $X$ is no longer the reward/payoff process. It is now the price process from which the reward/payoff $\phi(X)$ is computed. We shall also use the notation $X_{t}^{0, X_{0}}$ for $X_{t}$ whenever we need to emphasize the dependence of the process $X$ upon its initial condition. We now define the value function of the perpetual swing option problem with $\ell$ exercise rights and refraction time $\delta>0$ by:

$$
v^{(\ell)}\left(X_{0}\right)=\sup _{\left(\tau_{1}, \ldots, \tau_{\ell}\right) \in \mathcal{S}^{(\ell)}} \mathbb{E}\left\{\sum_{i=1}^{\ell} e^{-r \tau_{i}} \phi\left(X_{\tau_{i}}\right)\right\} .
$$

where the set $\mathcal{S}^{(\ell)}$ is defined by:

$$
\mathcal{S}^{(\ell)}=\left\{\vec{\tau}=\left(\tau_{1}, \ldots, \tau_{\ell}\right) \in \mathcal{S}^{\ell} ; \tau_{i}-\tau_{i-1} \geq \delta \text { for all } i=2, \ldots, \ell\right\},
$$

and $\mathcal{S}$ is the subset of $\mathcal{S}_{\infty}$ of all finite stopping times with values in $\mathbb{R}_{+}$. In the following, we restrict ourselves to the reward function

$$
\phi(x)=(K-x)^{+} \text {for some given parameter } K>0,
$$


so that our analysis of the optimal exercise of the swing option extends to the multiple exercise case, classical results proven in the case of an American put option with only one exercise right.

3.2. Perpetual American Puts. When $\ell=1$ the refracting time parameter $\delta$ is irrelevant. The following explicit solution of the perpetual American put option is well-known. We state it to introduce the notation we need in the sequel.

Theorem 2. The perpetual American put option value function is given by

$$
v^{(1)}(x)=\left(K-x \wedge x_{1}^{*}\right)\left[1 \wedge\left(\frac{x_{1}^{*}}{x}\right)^{\gamma}\right]
$$

where

$$
\gamma=\frac{2 r}{\sigma^{2}} \text { and } x_{1}^{*}=\frac{K \gamma}{1+\gamma} .
$$

The above result states in particular that

$$
v^{(1)}(x)=K-x=\phi(x) \text { if and only if } x \leq x_{1}^{*} .
$$

The perpetual American put option should be exercised whenever the price process $X$ is below the level $x_{1}^{*}$. In view of this, the interval $\left[0, x_{1}^{*}\right]$ is called the exercise region, and its complement $\left(x_{1}^{*}, \infty\right)$ is called the the continuation region.

In the subsequent paragraphs, we provide an extension of Theorem 2 to the case of perpetual options with multiple exercises. We first prove the existence of a non-empty and connected exercise region $\left[0, x_{\ell}^{*}\right]$ for the optimal multiple stopping problem with value function $v^{(\ell)}$. This region contains the exercise region $\left[0, x_{1}^{*}\right]$ of the perpetual American put option. We then provide a recursive characterization of the boundary $x_{\ell}^{*}$ as the unique zero of some functional involving the value function $v^{(\ell-1)}$. As a by-product, this characterization produces a quasi-constructive solution of the problem of interest, and allows us to prove that the sequence $\left(x_{\ell}^{*}\right)_{\ell}$ is increasing.

3.3. Single Stopping Time Formulation and ODE Characterization. We first apply the general characterization result of Theorem 1 to reduce the multiple stopping problem to a cascade of $p$ optimal single stopping problems. So we define inductively the value functions $v^{(k)}$ and the reward/payoff functions $\phi^{(k)}$ by setting:

$$
v^{(k)}(x)=\sup _{\tau \in \mathcal{S}} \mathbb{E}\left\{e^{-r \tau} \phi^{(k)}\left(X_{\tau}^{0, x}\right)\right\}
$$

where

$$
\phi^{(k)}(x)=\phi(x)+e^{-r \delta} \mathbb{E}\left\{v^{(k-1)}\left(X_{\delta}^{0, x}\right)\right\},
$$

i.e. the process $\left\{e^{-r t} v^{(k)}\left(X_{t}\right)\right\}$ is the continuous-time Snell envelope of the process $\left\{e^{-r t} \phi^{(k)}\left(X_{t}\right)\right\}$.

One can then write the dynamic programming principle for the single stopping time problem value function (19), and derive the so-called Hamilton-Jacobi-Bellman equation for $v^{(k)}$. Using classical analysis arguments, one can prove that this function is the unique continuous viscosity solution of the variational inequality

$$
\min \left\{-\mathcal{L} v^{(k)}, v^{(k)}-\phi^{(k)}\right\} \geq 0,
$$


where $\mathcal{L}$ is the linear second order differential operator

$$
\mathcal{L} \chi(x)=\frac{1}{2} \sigma^{2} x^{2} \frac{\partial^{2} \chi}{\partial x^{2}}(x)+r x \frac{\partial \chi}{\partial x}(x)-r \chi(x) .
$$

We shall not provide a proof of this claim because we do not use the above PDE characterization of $v^{(k)}$ in what follows.

Since $\phi$ is bounded and $X$ is continuous, the reward process $\left\{e^{-r t} \phi\left(X_{t}\right)\right\}_{t \geq 0}$ satisfies the conditions (1) and (8), and we can apply the results of the previous section. For later use, we provide the following well-known result (which obviously holds for more general diffusions), which gives among other things, the fact that the reward functions $\phi^{(k)}$ are continuous.

Lemma 2. Let $f:[0, \infty) \longrightarrow \mathbb{R}$ be such that $\mathbb{E}\left\{\left|f\left(X_{t}\right)\right|^{2}\right\}<\infty$, and let us consider the function

$$
\chi(x)=\mathbb{E}\left\{f\left(X_{t}^{0, x}\right)\right\}, \quad x \geq 0 .
$$

Then $\chi$ is continuous on $[0, \infty)$ and continuously differentiable on $(0, \infty)$.

Proof. We sketch the proof for the sake of completeness. Observe that:

$$
\chi(x)=\int f\left(z e^{\left(r-\sigma^{2} / 2\right) t}\right) \frac{1}{z \sigma \sqrt{2 \pi t}} e^{-(\ln z-\ln x)^{2} / 2 \sigma^{2} t} d z .
$$

The dominated convergence theorem allows differentiation inside the expectation and the desired result follows.

3.4. First Properties of Perpetual Swing Options. We first prove that the exercise region for the optimal stopping problem (19) is not empty.

Lemma 3. Let $k \geq 1$ be a given integer and let $\phi(x)=(K-x)^{+}$. Then

$$
v^{(k)}(x)=\phi^{(k)}(x) \text { whenever } 0 \leq x \leq x_{1}^{*} .
$$

Proof. We only need to prove that $v^{(k)} \leq \phi^{(k)}$ on $\left[0, x_{1}^{*}\right]$ as the reverse inequality is trivial. By definition of $\phi^{(k)}$, we have

$$
\begin{aligned}
v^{(k)}(x) & =\sup _{\tau \in \mathcal{S}} \mathbb{E}\left\{e^{-r \tau} \phi\left(X_{\tau}\right)+e^{-r(\tau+\delta)} v^{(k-1)}\left(X_{\tau+\delta}\right)\right\} \\
& \leq v^{(1)}(x)+\sup _{\tau \in \mathcal{S}} \mathbb{E}\left\{e^{-r(\tau+\delta)} v^{(k-1)}\left(X_{\tau+\delta}\right)\right\} .
\end{aligned}
$$

Notice that $\mathbb{E}\left\{e^{-r(\tau+\delta)} v^{(k-1)}\left(X_{\tau+\delta}\right)\right\} \leq \mathbb{E}\left\{e^{-r \delta} v^{(k-1)}\left(X_{\delta}\right)\right\}$ by the supermartingale property of the process $\left\{e^{-r t} v^{(k-1)}\left(X_{t}\right)\right\}_{t \geq 0}$. Since $v^{(1)}=\phi^{(1)}=\phi$ on $\left[0, x_{1}^{*}\right]$, this provides :

$$
v^{(k)}(x) \leq \phi(x)+\mathbb{E}\left\{e^{-r \delta} v^{(k-1)}\left(X_{\delta}\right)\right\}=\phi^{(k)}(x) \text { for } 0 \leq x \leq x_{1}^{*} .
$$

Our next result shows that the exercise region corresponding to the stopping problem (19) is connected, and defines the exercise boundaries $x_{k}^{*}$. 
Proposition 3. Let $k \geq 1$ be a given integer and as above, let us restrict ourselves to the payoff $\phi(x)=(K-x)^{+}$of the American put option. Then, there exists $x_{k}^{*} \in\left[x_{1}^{*}, K\right]$ such that

$$
v^{(k)}(x)=\phi^{(k)}(x) \text { if and only if } 0 \leq x \leq x_{k}^{*} .
$$

Proof. Let us assume to the contrary that the exercise region for the problem $v^{(k)}$ is not connected, and we will establish a contradiction. Let $0 \leq x_{1}<x_{2}$ be such that

$$
v^{(k)}\left(x_{i}\right)=\phi^{(k)}\left(x_{i}\right) \text { while } v^{(k)}>\phi^{(k)} \text { on }\left(x_{1}, x_{2}\right) .
$$

Since $\phi(x)=0$ for $x \geq K$, it follows that $x_{2} \leq K$, and therefore

$$
\phi(x)=K-x \text { for } x \in\left[x_{1}, x_{2}\right] .
$$

Setting

$$
\hat{x}=\frac{x_{1}+x_{2}}{2}, \quad S_{x_{i}}=\inf \left\{t \geq 0 ; X_{t}^{0, \hat{x}}=x_{i}\right\}
$$

it follows from the classical optimal stopping theory that the stopping time $\hat{S}=S_{x_{1}} \wedge S_{x_{2}}$ is optimal for the problem $v^{(k)}(\hat{x})$. See Subsection 2.1. By (23), we then see that :

$$
\begin{aligned}
v^{(k)}(\hat{x}) & =\mathbb{E}\left\{e^{-r \hat{S}} \phi\left(X_{\hat{S}}^{0, \hat{x}}\right)\right\}+\mathbb{E}\left\{e^{-r(\hat{S}+\delta)} v^{(k-1)}\left(X_{\hat{S}+\delta}^{0, \hat{x}}\right)\right\} \\
& =\phi\left(\mathbb{E}\left\{e^{-r \hat{S}} X_{\hat{S}}^{0, \hat{x}}\right\}\right)+\mathbb{E}\left\{e^{-r(\hat{S}+\delta)} v^{(k-1)}\left(X_{\hat{S}+\delta}^{0, \hat{x}}\right)\right\} \\
& =\phi(\hat{x})+\mathbb{E}\left\{e^{-r(\hat{S}+\delta)} v^{(k-1)}\left(X_{\hat{S}+\delta}^{0, \hat{x}}\right)\right\} \\
& \leq \phi(\hat{x})+\mathbb{E}\left\{e^{-r \delta} v^{(k-1)}\left(X_{\delta}^{0, \hat{x}}\right)\right\}=\phi^{(k)}(\hat{x}),
\end{aligned}
$$

where the last inequality follows from the super-martingale property of $\left\{e^{-r t} v^{(k-1)}\left(X_{t}^{0, \hat{x}}\right)\right\}_{t \geq 0}$. This proves that $v^{(k)}(\hat{x})=\phi^{(k)}(\hat{x})$ contradicting (22).

In view of this result, we conclude that the stopping time:

$$
\theta_{k}^{*}=\inf \left\{t \geq 0 ; X_{t} \leq x_{k}^{*}\right\}
$$

defines an optimal stopping rule for the problem (19), and that the value function of the problem is given by:

$$
v^{(k)}(x)=\mathbb{E}\left\{e^{-r \theta_{k}^{*}} \phi^{(k)}\left(X_{\theta_{k}^{*}}\right)\right\}=\phi^{(k)}\left(x \wedge x_{k}^{*}\right) \mathbb{E}\left\{e^{-r \theta_{k}^{*}}\right\}
$$

by the continuity of the process $X$.

3.5. Characterization of the Exercise Boundaries of Perpetual Put Swing Options. So far, we have proved that the multiple stopping problem given by the value function $v^{(\ell)}$ could be reduced to a cascade of single optimal stopping problems with value functions $v^{(k)}, k \leq \ell$ in such a way that the optimal stopping/exercise rules were given by the exit times of the intervals $\left[x_{k}^{*}, \infty\right)$. It is therefore natural to introduce for each (reward) function $\psi$ the function $w[\psi]$ defined by:

$$
w[\psi](x, b):=\mathbb{E}\left\{e^{-r S_{b}^{x}} \psi\left(X_{S_{b}^{x}}^{0, x}\right)\right\} \quad \text { where } S_{b}^{x}=\inf \left\{t \geq 0 ; X_{t}^{0, x} \leq b\right\},
$$


which we will use for $\psi=\phi^{(k)}$, and we make the following important observation:

$$
v^{(k)}(x)=\left\{\begin{array}{l}
\phi^{(k)}(x) \text { for } x \leq x_{k}^{*} \\
w\left[\phi^{(k)}\right]\left(x, x_{k}^{*}\right)=\max _{b \leq x} w\left[\phi^{(k)}\right](x, b) \text { for } x \geq x_{k}^{*} .
\end{array}\right.
$$

In order to compute explicitly the function $w\left[\phi^{(k)}\right]$, we use the following well-known property of the Brownian motion. For each $b \geq 0$, let us define by:

$$
T_{b}=\inf \left\{t \geq 0 ; \mu t+W_{t}=b\right\}
$$

the first hitting time of the barrier $b$ by the Brownian motion with drift $\mu$. It is well-known that the Laplace transform of $T_{b}$ is given by

$$
\mathbb{E}\left\{e^{-\lambda T_{b}}\right\}=e^{\mu b-|b| \sqrt{\mu^{2}+2 \lambda}} .
$$

Let

$$
\mu=\frac{\sigma}{2}(\gamma-1) \text { and } \beta(b, x)=\frac{1}{\sigma} \ln \left(\frac{b}{x}\right) .
$$

Then $S_{b}^{x}=T_{\beta(b, x)} \mathbf{1}_{\{x \geq b\}}$ and it follows from(29) that for any bounded measurable function $\psi: \mathbb{R}_{+} \longrightarrow$ $\mathbb{R}$, we have

$$
\begin{aligned}
w[\psi](x, b) & =\mathbb{E}\left\{e^{-r S_{b}^{x}} \psi\left(X_{S_{b}^{x}}^{0, x}\right)\right\} \\
& =\psi(x) \mathbf{1}_{\{x \leq b\}}+\psi(b) \mathbb{E}\left\{e^{-r T_{\beta(b, x)}}\right\} \mathbf{1}_{\{x>b\}} \\
& =\psi(x \wedge b)\left[1 \wedge\left(\frac{b}{x}\right)^{\gamma}\right] .
\end{aligned}
$$

Plugging this equality in (27) and recalling that $x_{k}^{*} \leq K$, we see that

$$
v^{(k)}(x)=\left\{\begin{array}{l}
K-x+e^{-r \delta} \mathbb{E}\left\{v^{(k-1)}\left(X_{\delta}^{0, x}\right)\right\} \text { for } x \leq x_{k}^{*} \\
x^{-\gamma} \max _{b \leq K} b^{\gamma}\left(K-b+e^{-r \delta} \mathbb{E}\left\{v^{(k-1)}\left(X_{\delta}^{0, b}\right)\right\}\right) \text { for } x \geq x_{k}^{*} .
\end{array}\right.
$$

In particular, if we use Lemma 2, this shows that the function $v^{(k)}$ is differentiable on $\left(0, x_{k}^{*}\right) \cup$ $\left(x_{k}^{*}, \infty\right)$. Since $0<x_{k}^{*}<K$, the boundary $x_{k}^{*}$ is the solution of the equation given by the first order condition for the above maximization problem. For the statement of this result, it is convenient to introduce the function

$$
u^{(k)}(x)=(1+\gamma)^{-1}\left[\gamma v^{(k)}(x)+x \frac{d}{d x} v^{(k)}(x)\right], x>0 .
$$

Observe that $v^{(k)}$ can be recovered from $u^{(k)}$ by

$$
v^{(k)}(x)=(1+\gamma) x^{-\gamma} \int_{0}^{x} y^{\gamma-1} u^{(k)}(y) d y
$$

and $v^{(k)}(0)=\phi(0) \sum_{i=0}^{k-1} e^{-i r \delta}$, as 0 is an absorbing boundary for the process $X$.

Lemma 4. For each integer $k \geq 1$, the function $u^{(k)}$ is non-increasing and continuous. Moreover the sequence $\left\{u^{(k)}\right\}_{k}$ is characterized by the induction formula:

$$
u^{(k)}(x)=\mathbf{1}_{\left\{x \leq x_{k}^{*}\right\}}\left(x_{1}^{*}-x+e^{-r \delta} \mathbb{E}\left\{u^{(k-1)}\left(X_{\delta}^{0, x}\right)\right\}\right) \text { and } u^{(0)}=0 .
$$


Furthermore, the boundary $x_{k}^{*}$ is uniquely defined by the equation:

$$
x_{1}^{*}-x_{k}^{*}+e^{-r \delta} \mathbb{E}\left\{u^{(k-1)}\left(X_{\delta}^{0, x_{k}^{*}}\right)\right\}=0 .
$$

Proof. The first order condition (35) is obtained by direct differentiation. In order to prove (34), we use the expression of $v^{(k)}$ given in (31). First, for $x \geq x_{k}^{*}$, it is clear that $u^{(k)}(x)=0$. Next, since $X_{\delta}^{0, x}=x X_{\delta}^{0,1}$, we see immediately that $u^{(k)}(x)=x_{1}^{*}-x+e^{-r \delta} \mathbb{E}\left\{u^{(k-1)}\left(X_{\delta}^{0, x}\right)\right\}$ for $x<x_{k}^{*}$.

The function $u^{(k)}$ is clearly continuous away from the point $x_{k}^{*}$. Recall Lemma 2. Using the first order condition (35), we see that $u^{(k)}\left(x_{k}^{*}-\right)=u^{(k)}\left(x_{k}^{*}+\right)=0$.

We now prove by induction that $u^{(k)}$ is non-increasing. This property holds trivially for $u^{(0)} \equiv 0$. Assume that $u^{(k-1)}$ is non-decreasing for some $k \geq 2$, then since $X_{\delta}^{0, x}$ is increasing in $x$, it follows from (34) that $u^{(k)}$ is non-increasing. Finally, we observe that the uniqueness of the exercise boundary $x_{k}^{*}$ follows from the fact that the function $u^{(k)}$ is non-increasing.

Remark 2. Formula (34) is particularly well suited for numerical computations. Indeed it is plain to evaluate the expectation by a simple Monte Carlo method. We used this remark to produce the numerical results reported Section 5. See Figures 1,2 and 3 for plots of the functions $u^{(k)}$.

Recall that Proposition 3 says that $x_{k}^{*} \geq x_{1}^{*}$. It is natural to expect that these exercise boundaries form a monotone sequence. We establish this highly expected increasing property of the sequence $\left(x_{k}^{*}\right)_{k \geq 1}$ in the present context.

Lemma 5. (i) The sequence $\left(u^{(k)}\right)_{k>0}$ is increasing, i.e. $u^{(k)} \geq u^{(k-1)}$ and $u^{(k)} \neq u^{(k-1)}$. (ii) The sequence $\left(x_{k}^{*}\right)_{k \geq 1}$ is strictly increasing.

Proof. Define the functions $\ell(x)=x-x_{1}^{*}$ and $g^{(k)}(x)=e^{-r \delta} \mathbb{E}\left\{u^{(k)}\left(X_{\delta}^{0, x}\right)\right\}$. We first prove that (ii) is a direct consequence of (i). Indeed, it follows from (i) that $g^{(k)}(x)>g^{(k-1)}(x)$ for all $x>0$. Since $x_{k}^{*}$ is the unique intersection point of the graphs of the functions $\ell$ and $g^{(k-1)}$, we conclude that $x_{k}^{*}>x_{k-1}^{*}$.

We prove (i) by an induction argument. We first remark that $u^{(1)}(x)=\left(x_{1}^{*}-x\right)^{+}$for all $x \geq 0$. Since $u^{(0)} \equiv 0$, this shows that $u^{(1)} \geq u^{(0)}$ and $u^{(1)} \neq u^{(0)}$. We next assume that $u^{(k-1)} \geq u^{(k-2)}$ and that $u^{(k-1)} \neq u^{(k-2)}$. Observe that this implies that $x_{k}^{*} \geq x_{k-1}^{*}$ by the first part of this proof. Moreover

- For $x>x_{k}^{*}$, we have $u^{(k)}(x)=u^{(k-1)}(x)=0$.

- For $x<x_{k-1}^{*}$, we have

$$
\left[u^{(k)}-u^{(k-1)}\right](x)=e^{-r \delta} \mathbb{E}\left\{\left[u^{(k-1)}-u^{(k-2)}\right]\left(X_{\delta}^{0, x}\right)\right\}>0 .
$$

- For $x \in\left[x_{k-1}^{*}, x_{k}^{*}\right]$, we have $u^{(k-1)}(x)=0$. Furthermore, since $u^{(k)}$ is non-increasing and $u^{(k)}\left(x_{k}^{*}\right)=0$, it follows that $u^{(k)}(x) \geq 0$. 


\section{Perpetual Put Swing Options with Infinitely Many Exercise Rights in the BLACK-SCHOLES MODEL}

In this section we study the asymptotic regime obtained when the number of exercise rights increases without bound. In order to do so, we analyze the value function:

$$
v^{(\infty)}\left(X_{0}\right)=\sup _{\left(\tau_{n}\right)_{n \geq 1} \in \mathcal{S}(\infty)} \mathbb{E}\left\{\sum_{n \geq 1} e^{-r \tau_{n}} \phi\left(X_{\tau_{n}}\right)\right\}
$$

where as before $\phi$ is the payoff $\phi(x)=(K-x)^{+}$of an American put option and where the set $\mathcal{S}^{(\infty)}$ of sequences of stopping times depends upon the refraction parameter $\delta>0$ in the following way:

$$
\mathcal{S}^{(\infty)}=\left\{\left(\tau_{n}\right)_{n \geq 1} \in \mathcal{S}^{\mathbb{N}} ; \tau_{n+1}-\tau_{n} \geq \delta \text { for all } n \geq 1\right\} .
$$

Observe that, since $\phi \leq K$, we have for all $\left(\tau_{n}\right)_{n \geq 1} \in \mathcal{S}^{(\infty)}$ that:

$$
\sum_{n \geq 1} e^{-r \tau_{n}} \phi\left(X_{\tau_{n}}\right) \leq K \sum_{n \geq 1} e^{-r \tau_{n}} \leq K \sum_{n \geq 0} e^{-r n \delta}
$$

Therefore:

$$
v^{(\infty)}\left(X_{0}\right) \leq K\left(1-e^{-r \delta}\right)^{-1}
$$

We recast the current problem in the framework of an optimal single stopping problem.

Proposition 4. The value function $v^{(\infty)}$ satisfies:

(38) $v^{(\infty)}\left(X_{0}\right)=\sup _{\tau \in \mathcal{S}} \mathbb{E}\left\{\phi^{(\infty)}\left(X_{\tau}\right)\right\}$ where $\phi^{(\infty)}(x)=\phi(x)+e^{-r \delta} \mathbb{E}\left\{v^{(\infty)}\left(X_{\delta}^{0, x}\right)\right\}$.

Proof. 1. Let $\tau \in \mathcal{S}$ and $\left(\bar{\tau}_{i}\right)_{i \geq 1} \in \mathcal{S}^{(\infty)}$, and let us define a new sequence $\left\{\tau_{i}\right\}_{i \geq 1}$ of stopping times by $\tau_{1}=\tau, \tau_{i}=\tau+\delta+\bar{\tau}_{i+1}$. Then $\left(\tau_{i}\right)_{i \geq 1} \in \mathcal{S}^{(\infty)}$. Therefore

$$
\begin{aligned}
v^{(\infty)}\left(X_{0}\right) & \geq \mathbb{E}\left\{\sum_{i \geq 1} e^{-r \tau_{i}} \phi\left(X_{\tau_{i}}\right)\right\} \\
& =\mathbb{E}\left\{e^{-r \tau} \phi\left(X_{\tau}\right)+e^{-r(\tau+\delta)} \mathbb{E}\left\{\sum_{i \geq 1} e^{-r \bar{\tau}_{i}} \phi\left(X_{\tau+\delta+\bar{\tau}_{i}}\right) \mid \mathcal{F}_{\tau+\delta}\right\}\right\} .
\end{aligned}
$$

Since the sequence $\left\{\bar{\tau}_{i}\right\}_{i \geq 1} \in \mathcal{S}^{(\infty)}$ and the stopping time $\tau \in \mathcal{S}$ were arbitrary, this gives the inequality:

$$
v^{(\infty)}\left(X_{0}\right) \geq \sup _{\tau \in \mathcal{S}} \mathbb{E}\left\{e^{-r \tau} \phi\left(X_{\tau}\right)+e^{-r(\tau+\delta)} v^{(\infty)}\left(X_{\tau+\delta}\right)\right\}
$$


2. In order to establish the reverse inequality, we pick $\varepsilon>0$ and an $\varepsilon$-optimal stopping rule $\left\{\hat{\tau}_{i}\right\}_{i \geq 1} \in$ $\mathcal{S}^{(\infty)}$, i.e.

$$
\begin{aligned}
v^{(\infty)}\left(X_{0}\right)-\varepsilon & \leq \mathbb{E}\left\{\sum_{i \geq 1} e^{-r \hat{\tau}_{i}} \phi\left(X_{\hat{\tau}_{i}}\right)\right\} \\
& =\mathbb{E}\left\{e^{-r \hat{\tau}_{1}} \phi\left(X_{\hat{\tau}_{1}}\right)+e^{-r\left(\hat{\tau}_{1}+\delta\right)} \sum_{i \geq 1} e^{-r \bar{\tau}_{i}} \phi\left(X_{\hat{\tau}_{1}+\delta+\bar{\tau}_{i}}\right)\right\},
\end{aligned}
$$

where we used the stopping times $\bar{\tau}_{i}$ defined by:

$$
\bar{\tau}_{i}=\hat{\tau}_{i+1}-\hat{\tau}_{1}-\delta \text { for } i \geq 1 \text {. }
$$

We now observe that $\bar{\tau}_{1} \geq 0$ and $\bar{\tau}_{i+1}-\bar{\tau}_{i} \geq \delta$ a.s. Furthermore, considering the shifted filtration $\mathbb{F}^{\hat{\tau}_{1}+\delta}=\left\{\mathcal{F}_{t}^{\hat{\tau}_{1}+\delta}\right\}_{t \geq 0}$, we see that $\bar{\tau}_{i}$ is an $\mathbb{F}^{\hat{\tau}_{1}+\delta}$-stopping time, and therefore

$$
\mathbb{E}\left\{\sum_{i \geq 1} e^{-r \bar{\tau}_{i}} \phi\left(X_{\hat{\tau}_{1}+\delta+\bar{\tau}_{i}}\right) \mid \mathcal{F}_{\hat{\tau}_{1}+\delta}\right\} \leq v^{(\infty)}\left(X_{\hat{\tau}_{1}+\delta}\right),
$$

by definition of the value function $v^{(\infty)}$. Plugging this inequality in (39), we deduce that

$$
\begin{aligned}
v^{(\infty)}\left(X_{0}\right)-\varepsilon & \leq \mathbb{E}\left\{e^{-r \hat{\tau}_{1}} \phi\left(X_{\hat{\tau}_{i}}\right)+e^{-r\left(\hat{\tau}_{1}+\delta\right)} v^{(\infty)}\left(X_{\hat{\tau}_{1}+\delta}\right)\right\} \\
& \leq \sup _{\tau \in \mathcal{S}} \mathbb{E}\left\{e^{-r \tau} \phi\left(X_{\tau}\right)+e^{-r(\tau+\delta)} v^{(\infty)}\left(X_{\tau+\delta}\right)\right\}
\end{aligned}
$$

Next, we proceed exactly as in the proofs of Lemma 3 and Proposition 3 to obtain the following result.

Proposition 5. There exists $x_{\infty}^{*} \in\left[x_{1}^{*}, K\right]$ such that

$$
v^{(\infty)}(x)=\phi^{(\infty)}(x) \text { if and only if } 0 \leq x \leq x_{\infty}^{*} .
$$

Consequently, the stopping time

$$
\theta_{\infty}^{*}=\inf \left\{t \geq 0: X_{t} \leq x_{\infty}^{*}\right\}
$$

defines an optimal stopping rule for the problem (36). In order to characterize further the boundary $x_{\infty}^{*}$, we proceed as in the previous section by observing that

$$
v^{(\infty)}(x)=\left\{\begin{array}{l}
\phi^{(\infty)}(x) \text { for } x \leq x_{\infty}^{*} \\
w\left[\phi^{(\infty)}\right]\left(x, x_{\infty}^{*}\right)=\max _{b \leq x} w\left[\phi^{(\infty)}\right](x, b) \text { for } x \geq x_{\infty}^{*}
\end{array}\right.
$$

which provides by the same computations:

$$
v^{(\infty)}(x)=\left\{\begin{array}{l}
K-x+e^{-r \delta} \mathbb{E}\left\{v^{(\infty)}\left(X_{\delta}^{0, x}\right)\right\} \text { for } x \leq x_{\infty}^{*} \\
x^{-\gamma} \max _{b \leq K} b^{\gamma}\left(K-b+e^{-r \delta} \mathbb{E}\left\{v^{(\infty)}\left(X_{\delta}^{0, b}\right)\right\}\right) \text { for } x \geq x_{\infty}^{*}
\end{array}\right.
$$


Recall from (37) that $v^{(\infty)}$ is bounded. Then the function $x \longmapsto \mathbb{E}\left\{v^{(\infty)}\left(X_{\delta}^{0, x}\right)\right\}$ is differentiable by Lemma 2. Therefore, we deduce from (41) that $v^{(\infty)}$ is differentiable on $\left[0, x_{\infty}^{*}\right) \cup\left(x_{\infty}^{*}, \infty\right)$. Since $0<x_{\infty}^{*}<K$, the boundary $x_{\infty}^{*}$ solves the first order condition of the above maximization problem. As in the previous section, it is convenient to introduce the function:

$$
u^{(\infty)}(x)=(1+\gamma)^{-1}\left[\gamma v^{(\infty)}(x)+x \frac{d}{d x} v^{(\infty)}(x)\right], \quad x>0
$$

and we observe that $v^{(\infty)}$ can be recovered from $u^{(\infty)}$ by

$$
\begin{aligned}
v^{(\infty)}(x) & =v^{(\infty)}(0)+(1+\gamma) x^{-\gamma} \int_{0}^{x} y^{\gamma-1} u^{(k)}(y) d y \\
& =K\left(1-e^{-r \delta}\right)^{-1}+(1+\gamma) x^{-\gamma} \int_{0}^{x} y^{\gamma-1} u^{(\infty)}(y) d y
\end{aligned}
$$

We now state the first order conditions for the optimality of $x_{\infty}^{*}$.

Lemma 6. The function $u^{(\infty)}$ is continuous and satisfies

$$
u^{(\infty)}(x)=\mathbf{1}_{\left\{x \leq x_{\infty}^{*}\right\}}\left(x_{1}^{*}-x+e^{-r \delta} \mathbb{E}\left\{u^{(\infty)}\left(X_{\delta}^{0, x}\right)\right\}\right),
$$

and the boundary $x_{\infty}^{*}$ solves

$$
x_{1}^{*}-x_{\infty}^{*}+e^{-r \delta} \mathbb{E}\left\{u^{(\infty)}\left(X_{\delta}^{0, x_{\infty}^{*}}\right)\right\}=0 .
$$

Proof. Except the continuity of $u^{(\infty)}$, all claims follow by the same arguments as in the proof of Lemma 4. Now, since $v^{(\infty)}$ is bounded by (37), observe that the function $x \longmapsto \mathbb{E}\left\{v^{(\infty)}\left(X_{\delta}^{0, x}\right)\right\}$ is continuously differentiable. By (41), this shows that $v^{(\infty)}$ is continuously differentiable on $\left(0, x_{\infty}^{*}\right)$, implying the continuity of $u^{(\infty)}$ on this set. Since $u^{(\infty)}=0$ on $\left(x_{\infty}^{*}, \infty\right)$, it only remains to check the continuity of $u^{(\infty)}$ at the point $x_{\infty}^{*}$. This is a direct consequence of the first order condition (44).

We are now in a position to characterize the boundary $x_{\infty}^{*}$ in terms of the integer-valued random variable $N_{\delta}(b)$ defined as the first crossing time of the geometric Brownian motion samples separated by the refracting period, i.e. the integer:

$$
N_{\delta}(b):=\min \left\{n \geq 1 ; X_{n \delta}^{0,1}>b\right\} .
$$

Proposition 6. The function $u^{(\infty)}$ is given by:

$$
u^{(\infty)}(x)=\mathbb{E}\left\{\sum_{n=0}^{N_{\delta}\left(x_{\infty}^{*} / x\right)-1} e^{-n r \delta}\left(x_{1}^{*}-X_{n \delta}^{0, x}\right)\right\},
$$

and the the optimal exercise boundary for the problem (36) is :

$$
x_{\infty}^{*}=x_{1}^{*} \frac{\mathbb{E}\left\{\sum_{n<N_{\delta}(1)} e^{-n r \delta}\right\}}{\mathbb{E}\left\{\sum_{n<N_{\delta}(1)} e^{-n r \delta} X_{n \delta}^{0,1}\right\}} .
$$


Proof. Iterating (43), and observing that $\prod_{i=0}^{j} \mathbf{1}_{\left\{X_{i \delta}^{0, x} \leq x_{1}^{*}\right\}}=\mathbf{1}_{\left\{j<N_{\delta}\left(x_{\infty}^{*} / x\right)\right\}}$, we see that

$$
\begin{aligned}
u^{(\infty)}(x)= & \mathbb{E}\left\{\sum_{j=0}^{n} e^{-j r \delta}\left(x_{1}^{*}-X_{j \delta}^{0, x}\right) \mathbf{1}_{\left\{j<N_{\delta}\left(x_{\infty}^{*} / x\right)\right\}}\right\} \\
& +e^{-(n+1) r \delta} \mathbb{E}\left\{u^{(\infty)}\left(X_{(n+1) \delta}^{0, x}\right) \mathbf{1}_{\left\{n<N_{\delta}\left(x_{\infty}^{*} / x\right)\right\}}\right\},
\end{aligned}
$$

by definition of $N_{\delta}(b)$. Now, recall that $u^{(\infty)}=0$ outside the compact interval $\left[0, x_{\infty}^{*}\right]$ and that it is continuous by Lemma 6 . Then $u^{(\infty)}$ is bounded and

$$
\lim _{n \rightarrow \infty} e^{-(n+1) r \delta} \mathbb{E}\left\{u^{(\infty)}\left(X_{(n+1) \delta}^{0, x}\right) \mathbf{1}_{\left\{n<N_{\delta}\left(x_{\infty}^{*} / x\right)\right\}}\right\}=0 .
$$

By the dominated convergence theorem, this implies that :

$$
\begin{aligned}
u^{(\infty)}(x) & =\lim _{n \rightarrow \infty} \mathbb{E}\left\{\sum_{j=0}^{n} e^{-j r \delta}\left(x_{1}^{*}-X_{j \delta}^{0, x}\right) \mathbf{1}_{\left\{j<N_{\delta}\left(x_{\infty}^{*} / x\right)\right\}}\right\} \\
& =\mathbb{E}\left\{\sum_{j=0}^{\infty} e^{-j r \delta}\left(x_{1}^{*}-X_{j \delta}^{0, x}\right) \mathbf{1}_{\left\{j<N_{\delta}\left(x_{\infty}^{*} / x\right)\right\}}\right\},
\end{aligned}
$$

completing the proof of (46). We now obtain (47) by writing that $u^{(\infty)}\left(x_{\infty}^{*}\right)=0$ :

$$
\begin{aligned}
0 & =\mathbb{E}\left\{\sum_{n=0}^{N_{\delta}(1)-1} e^{-n r \delta}\left(x_{1}^{*}-x_{\infty}^{*} X_{n \delta}^{0,1}\right)\right\} \\
& =x_{1}^{*} \mathbb{E}\left\{\sum_{n=0}^{N_{\delta}(1)-1} e^{-n r \delta}\right\}-x_{\infty}^{*} \mathbb{E}\left\{\sum_{n=0}^{N_{\delta}(1)-1} e^{-n r \delta} X_{n \delta}^{0,1}\right\} .
\end{aligned}
$$

We conclude this section by proving the convergence of the solution of the perpetual swing option problem with finitely many rights to the corresponding solution when the number of exercise rights is infinite.

Proposition 7. We have the following convergence results:

$$
x_{k}^{*} \longrightarrow x_{\infty}^{*} \text { and }\left(u^{(k)}, v^{(k)}\right) \longrightarrow\left(u^{(\infty)}, v^{(\infty)}\right) \text { uniformly on }[0, \infty) \text {. }
$$

Proof. 1. We first prove the convergence of $\left\{x_{k}^{*}\right\}_{k \geq 1}$ and the uniform convergence of $\left\{u^{(k)}\right\}_{k \geq 1}$. By Lemma 5, the sequences $\left\{x_{k}^{*}\right\}_{k \geq 1}$ and $\left\{u^{(k)}\right\}_{k \geq 1}$ are increasing. Moreover $x_{k}^{*} \leq K$, and $u^{(k)} \leq$ $K\left[1-e^{-r \delta}\right]^{-1}$ as it can be immediately checked from (34). Then there exist $\bar{x} \leq K$ and $\bar{u}$ : $[0, \infty) \longrightarrow \mathbb{R}$ such that

$$
x_{k}^{*} \longrightarrow \bar{x} \text { and } u^{(k)}(x) \longrightarrow \bar{u}(x) \text { for all } x \geq 0 .
$$

By dominated convergence, it follows from (34) and (35) that

$$
\bar{u}(x)=\mathbf{1}_{x \leq \bar{x}}\left\{x_{1}^{*}-x+e^{-r \delta} \mathbb{E}\left\{\bar{u}\left(X_{\delta}^{0, x}\right)\right\}\right\}
$$


and

$$
x_{1}^{*}-\bar{x}+e^{-r \delta} \mathbb{E}\left\{\bar{u}\left(X_{\delta}^{0, \bar{x}}\right)\right\}=0 .
$$

Since $\bar{u}$ is bounded, this proves that $\bar{u}$ is continuous. Since, for each $k \geq 1, u^{(k)}=0$ outside the compact interval $[0, K]$, it then follows from Dini's theorem that $\left\{u^{(k)}\right\}_{k \geq 1}$ converges to $\bar{u}$ uniformly on $[0, \infty)$.

2. We now show that $(\bar{x}, \bar{u})=\left(x_{\infty}^{*}, u^{(\infty)}\right)$. To see this, notice that (50) and (51) show that $(\bar{x}, \bar{u})$ satisfy the conditions which have been established for $\left(x_{\infty}^{*}, u^{(\infty)}\right)$ in Lemma 6. Observing that the characterization of $\left(x_{\infty}^{*}, u^{(\infty)}\right)$ in Proposition 6 is obtained by means of these equations, we conclude that $(\bar{x}, \bar{u})=\left(x_{\infty}^{*}, u^{(\infty)}\right)$.

3. It remains to prove the uniform convergence of sequence $\left(v^{(k)}\right)_{k \geq 1}$ towards $v^{(\infty)}$. To see this, we directly compute by (33) and (42) that :

$$
\begin{aligned}
\left|v^{(k)}(x)-v^{(\infty)}(x)\right| & \leq\left|v^{(k)}(0)-v^{(\infty)}(0)\right|+(1+\gamma) x^{-\gamma} \int_{0}^{x} y^{\gamma-1}\left|u^{(k)}(y)-u^{(\infty)}(y)\right| d y \\
& \leq\left|v^{(k)}(0)-v^{(\infty)}(0)\right|+(1+\gamma)\left\|u^{(k)}-u^{(\infty)}\right\|_{\infty} x^{-\gamma} \int_{0}^{x} y^{\gamma-1} d y \\
& =\left|v^{(k)}(0)-v^{(\infty)}(0)\right|+\gamma^{-1}(1+\gamma)\left\|u^{(k)}-u^{(\infty)}\right\|_{\infty},
\end{aligned}
$$

where we used the notation $\|\cdot\|_{\infty}$ for the supremum norm of a function. The desired result then follows from the uniform convergence of $\left\{u^{(k)}\right\}_{k \geq 1}$ towards $u^{(\infty)}$.

\section{Numerical Results for the Infinite Maturity Problem}

This section contains a small sample of numerical results chosen to illustrate the theoretical results proven in this paper. The computations reported in this section use a strike price $K=1$, a refraction period $\delta=.01$, a volatility $\sigma=.35$, and a short interest rate $r=.04$. These give the values $\gamma=.653$ and $x_{1}^{*}=.395$.

We computed approximations of the function $u_{k}$ over a grid of 250 points $x$ regularly spaced between 0 and 1 . We used the values $1,2, \cdots, 250$ for $k$. The expectation appearing in the recursive formula (34) proven in Lemma 4 and giving the values of $u_{k}$ in terms of $u_{k-1}$ was computed from 20, 000 Monte Carlo random samples of the random variable $X_{\delta}$ starting from the values $X_{0}=x$ of the grid.

Figure 1 shows the graphs of the first 250 functions $u_{k}$ computed in this way. One clearly sees the fact that they are increasing. This growth in $k$ near the origin could appear in contradiction with the fact that the $u_{k}$ 's were uniformly bounded (i.e. the supremum norm of $u_{k}$ is bounded in $k$ ) which we proved in the text. So we plotted these supremum norms as functions of $k$.

Figure 2 is very much consistent with the uniform boundedness of the functions $u_{k}$.

This is confirmed by the surface plot given in Figure 3 which gives a different perspective on the same data. Finally, Figure 4 plots the values of $x_{k}^{*}$ as a function of $k$. After an early sharp increase, these thresholds level off rapidly toward their limiting value $x_{\infty}^{*}$. 


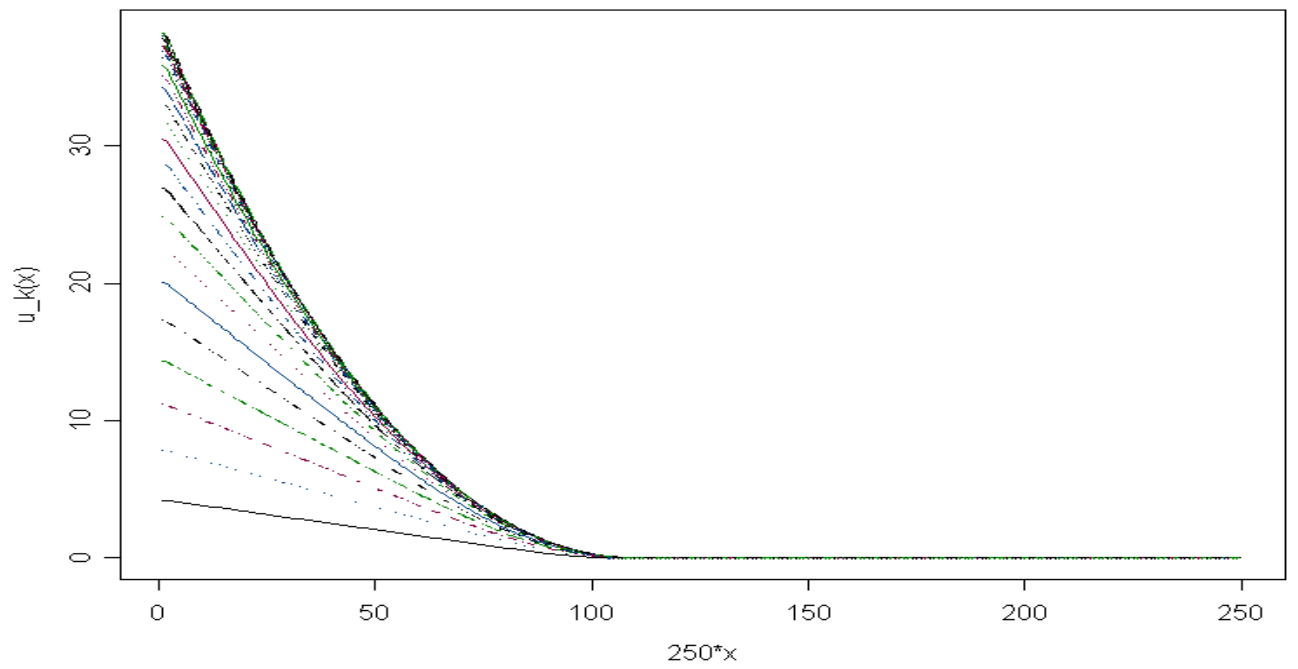

FIGURE 1. Time series plots of the graphs of the functions $u_{k}$ for $k=10 j$ with $j=1,2, \cdots, 25$. Note that the numerical value of $x_{\infty}^{*}$ would be .725 .

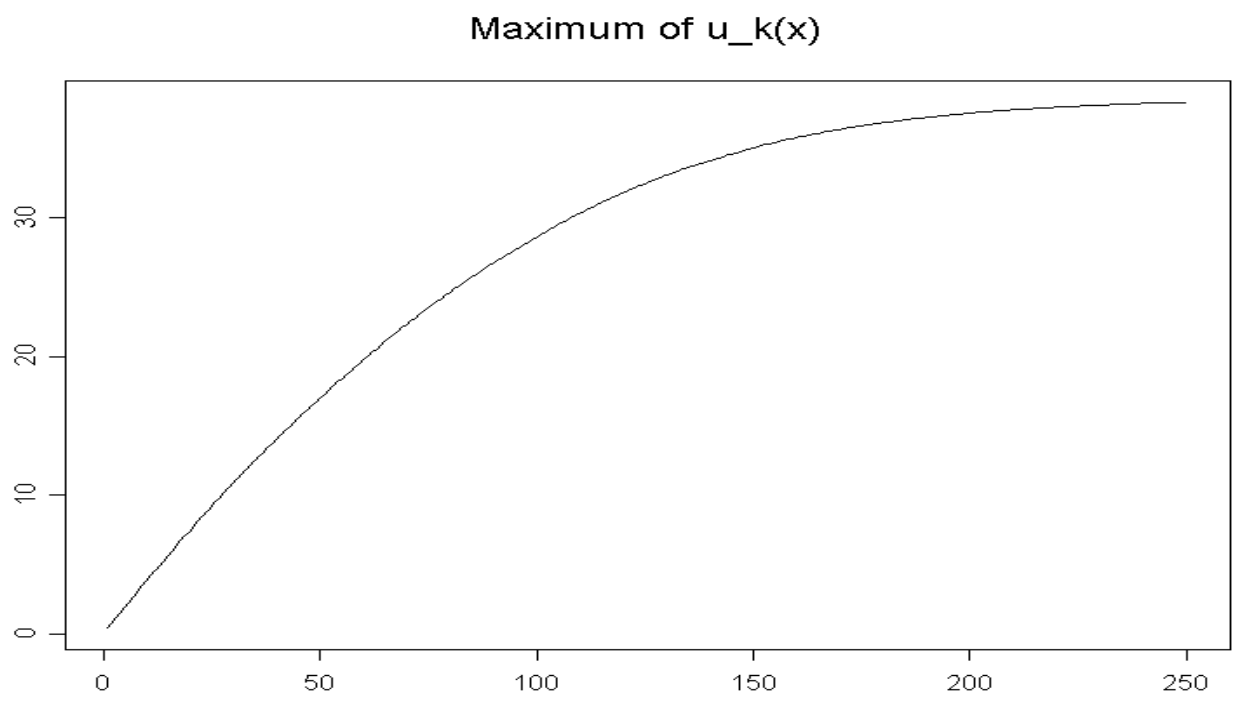

FIGURE 2. Time series plot of the values of the sup-norm of the function $u_{k}$ for $k=1,2, \cdots, 250$.

Figure 5 gives a surface plot of $x_{\infty}^{*}$ as a function of the the two free parameters $r \delta$ and $\sigma \sqrt{\delta} / 2$. Notice that $x_{\infty}^{*}$ is found to be decreasing in $\sigma \sqrt{\delta} / 2$ and increasing in $r \sqrt{\delta}$, both properties being very natural. 


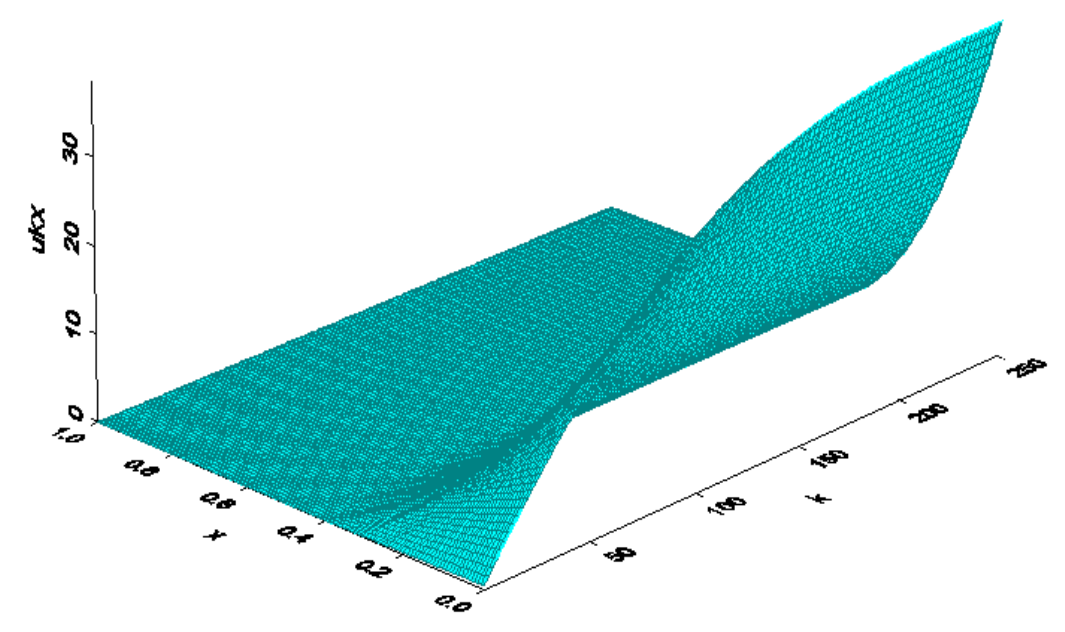

FigURE 3. Surface plot of the values $u_{k}(x)$ for $x \in[0,1]$ and for $k=1,2, \cdots, 250$..

\section{Numerical Results for the Finite Horizon Problem}

In this section, we return to the finite horizon multiple stopping problem, and we set

$$
T=1
$$

without any loss of generality. In other words we consider swing options with maturity one year. Our objective is to present and implement a Monte Carlo numerical procedure for the computation of the value function of the multiple stopping problem

$$
v^{(\ell)}\left(0, X_{0}\right):=\sup _{\vec{\tau} \in \overline{\mathcal{S}}^{(\ell)}} \sum_{i=1}^{\ell} \mathbb{E}\left\{e^{-r \tau_{i}} \phi\left(X_{\tau_{i}}\right)\right\},
$$

and the associated exercise region. As before, $X$ is the Black-Scholes price process defined in (16), $r$ is a constant instantaneous interest rate, and $\phi(x)=(K-x)^{+}$is the European put pay-off function with strike $K>0$.

6.1. Discrete Time Approximation. In order to estimate the value function $v^{(\ell)}$, we first need to define a convenient discrete-time approximation. For each integer $n \geq 1$, we introduce the partition $\mathbf{T}_{n}=\left\{t_{j}:=j / n\right\}_{0 \leq j \leq n}$ of the time interval $\mathbf{T}:=[0,1]$, and we use the notation $\mathbb{S}_{n}$ for the subset 


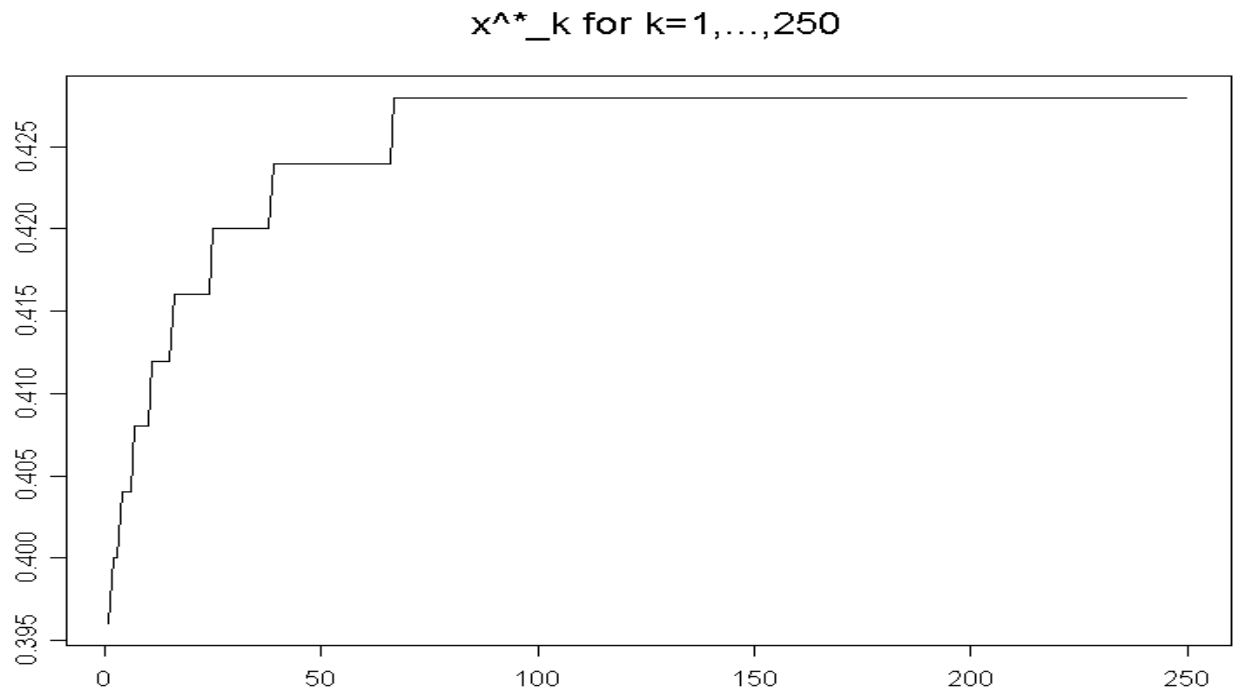

FIGURE 4. Plot of the values of $x_{k}^{*}$ computed for $k=1,2, \cdots, 250$.

of $\mathcal{S}_{1}$ defined by

$$
\mathbb{S}_{n}:=\left\{\tau \in \mathcal{S}_{1} ; \tau \in \mathbf{T}_{n} \text { a.s. }\right\} \text {. }
$$

We use the same notation as in Subsection 2.3. In particular, $Y^{(i)}$ stands for the Snell envelope of the reward process $\phi^{(i)}\left(t, X_{t}\right)$, where the reward function $\phi^{(i)}$ is defined inductively together with the successive value functions $v^{(i)}$ by:

$$
\phi^{(i)}(t, x):=\phi(x)+e^{-r \delta} \mathbb{E}\left\{v^{(i-1)}\left(t+\delta, X_{\delta}^{0, x}\right)\right\} \quad \text { for } \quad t \leq 1-\delta,
$$

and

$$
\phi^{(i)}(t, x):=\phi(x) \quad \text { for } \quad 1-\delta<t \leq 1,
$$

while the $v^{(i)}$ 's are given by:

$$
v^{(i)}(t, x)=\sup _{\tau \in \mathcal{S}_{t, T}} \mathbb{E}\left\{e^{-r \tau} \phi^{(i)}\left(\tau, X_{\tau}^{t, x}\right)\right\} .
$$

Recall the convention $v^{(0)} \equiv 0$. For each integer $n \geq 1$, we propose a natural discrete time approximation for the value function of the problem. It is given by

$$
v_{n}^{(i)}(t, x):=\sup _{\tau \in \mathbb{S}_{n} \cap \mathcal{S}_{t, T}} \mathbb{E}\left[e^{-r \tau} \phi_{n}^{(i)}\left(\tau, X_{\tau}^{t, x}\right)\right],
$$

starting as before with $v_{n}^{(0)} \equiv 0$, and where:

$$
\phi_{n}^{(i)}(t, x)=\phi(x)+e^{-r \delta} \mathbb{E}\left\{v_{n}^{(i-1)}\left(t+\delta, X_{\delta}^{0, x}\right)\right\} \quad \text { for } \quad t \leq 1-\delta,
$$




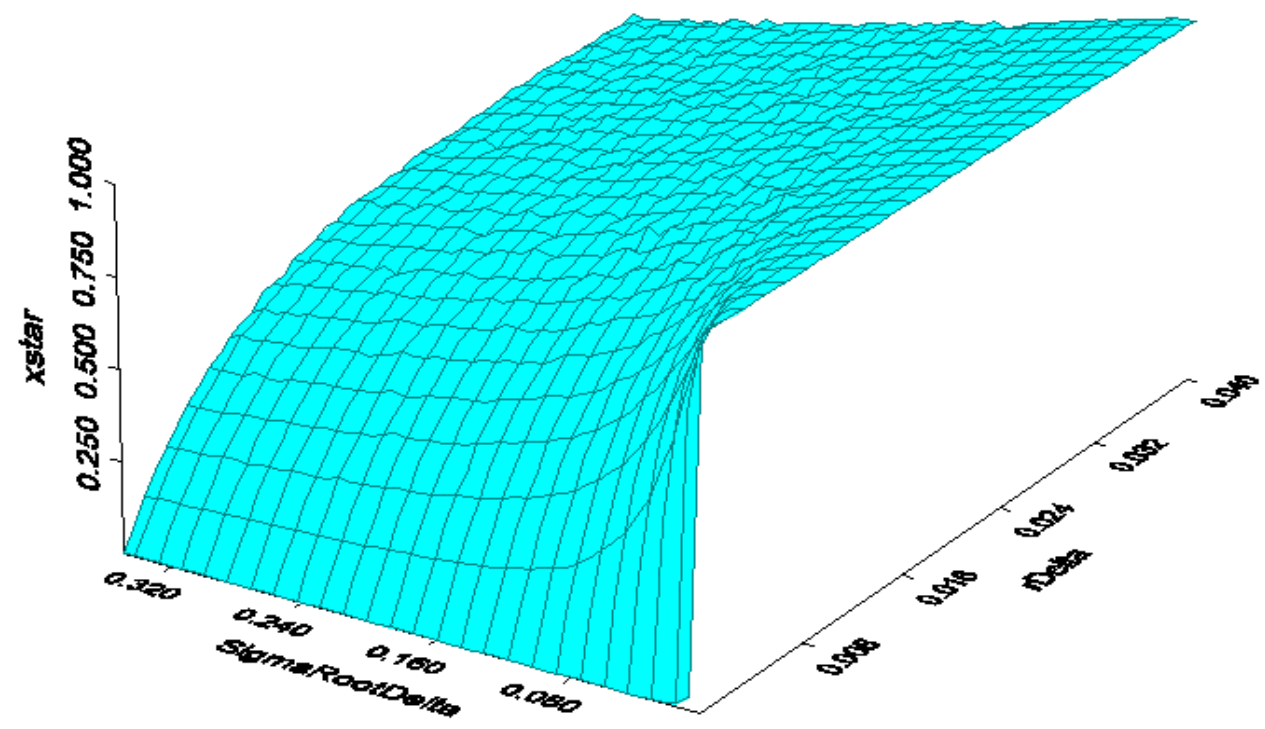

FIGURE 5. Surface plot of the values of $x_{\infty}^{*}$ as function of the two free parameters $r \delta$ and $\sigma \sqrt{\delta} / 2$.

and

$$
\phi_{n}^{(i)}(t, x)=\phi(x) \quad \text { for } \quad 1-\delta<t \leq 1 .
$$

In the discrete-time framework, it is well-known that the Snell envelope is easily computed by the backward induction

$$
v_{n}^{(i)}\left(t_{n}, X_{t_{n}}\right)=\phi^{(i)}\left(t_{n}, X_{t_{n}}\right)
$$

and

$$
v_{n}^{(i)}\left(t_{j-1}, X_{t_{j-1}}\right)=\max \left\{\phi_{n}^{(i)}\left(t_{j-1}, X_{t_{j-1}}\right) ; e^{-r / n} \mathbb{E}\left[v_{n}^{(i)}\left(t_{j}, X_{t_{j}}\right) \mid \mathcal{F}_{t_{j-1}}^{(n)}\right]\right\},
$$

where $\mathcal{F}_{t_{j}}^{(n)}=\sigma\left(X_{t_{k}}, k \leq j\right)$ is the discrete-time filtration. Since the process $X$ is Markov, the latter conditional expectation reduces to the computation of a regression function :

$$
\mathbb{E}\left\{v_{n}^{(i)}\left(t_{j}, X_{t_{j}}\right) \mid \mathcal{F}_{t_{j-1}}^{n}\right\}=\mathbb{E}\left\{v_{n}^{(i)}\left(t_{j}, X_{t_{j}}\right) \mid X_{t_{j-1}}\right\}=: \quad \rho_{n}^{(i)}\left(t_{j-1}, X_{t_{j-1}}\right) .
$$


6.2. Computation of the Conditional Expectations. As evidenced by the above formulae, the practical implementation of this backward procedure requires the computation of many conditional expectations, and the numerical implementations will vary according to the choice made for the evaluations of these regression functions. We briefly review the most obvious of these choices before concentrating on the method which we choose to develop.

Nonparametric Regression. At this stage of the analysis, many nonparametric regression procedures can be brought to bear, but we refrain from attempting to reviewing them all, and we restrict ourselves to a selected few.

(i) The Kernel Method. This well-known technique from non-parametric statistics, see e.g. Bosq [3], has been suggested in the context of the American put option by Carrière [8]. This method is based on the observation that for random variables or vectors $A$ and $B$ :

$$
\mathbb{E}\{A \mid B=b\}=\frac{\mathbb{E}\left\{A \delta_{b}(B)\right\}}{\mathbb{E}\left\{\delta_{b}(B)\right\}},
$$

where $\delta_{b}$ is the Dirac mass at the point $b$. Then, given an approximate identity, i.e. a family of functions $\kappa_{h}$ which converges to $\delta_{0}$ when $h \rightarrow 0$ (in some sense which we will not make precise here), it is natural to introduce the approximation

$$
\frac{\mathbb{E}\left\{A \kappa_{h}(B-b)\right\}}{\mathbb{E}\left\{\kappa_{h}(B-b)\right\}},
$$

and for a sample $\left(A^{(s)}, B^{(s)}\right)_{1 \leq s \leq N}$ of $N$ independent random vectors with the same distribution as $(A, B)$, the kernel estimator of the regression is given by:

$$
\hat{\mathbb{E}}\{A \mid B=b\}=\frac{\frac{1}{N} \sum_{s=1}^{N} A^{(s)} \kappa_{h_{N}}\left(B^{(s)}-b\right)}{\frac{1}{N} \sum_{s=1}^{N} A^{(s)} \kappa_{h_{N}}\left(B^{(s)}-b\right)},
$$

where $h_{N}$ is a sequence of positive numbers converging to zero. Despite the freedom to choose the rate of convergence of $h_{n}$ to 0 , the bias introduced by the approximation of the Dirac mass by the kernel function is responsible for the fact that the classical $\sqrt{N}$ rate of convergence of the central limit theorem is lost. We refer to [3] for a detailed analysis of the rate of convergence of the kernel estimator. There the interested reader will find extensions to dependent samples and proofs that this rate decreases dramatically when the dimension of the random variable $B$ increases.

(ii) The Basis Expansion Method. Let us assume momentarily that $B$ is a $p$-dimensional random vector, and let us denote by $\mu_{B}$ its distribution in $\mathbb{R}^{p}$. For any square integrable random variable $A$, the regression function $\mathbb{R}^{p} \ni b \hookrightarrow \mathbb{E}\{A \mid B=b\}$ can be viewed (and characterized) as an element of $L^{2}\left(\mathbb{R}^{p}, \mu_{B}\right)$. As such, it can be approximated by the partial sums of its decomposition on any orthonormal basis of this Hilbert space. Since the coefficients in such an expansion are expectations of products of $A$ by functions of $B$, they can be estimated from a random sample $\left(A^{(s)}, B^{(s)}\right)_{1 \leq s \leq N}$. This estimation technique is also standard in nonparametric regression. Its use in the context of American option pricing was suggested by Longstaff and Schwartz [20], and the corresponding price estimate has been shown to be consistent by Clément, Lamberton and Protter [9]. As in the case of the kernel method, the $\sqrt{N}$-rate of convergence is lost because of the bias introduced by the finite dimensional approximation. The choice of the orthonormal basis can drastically influence the rate of 
convergence. For example, it was shown by Egloff and Min-oo [11] that the rate of convergence of this algorithm could be exponentially slow. See for example their Theorem 6.15 .

Malliavin Calculus Based Simulation Method. The technique which we now consider has been proposed by Fournié, Lasry, Lebuchoux and Lions [13], and further developed by Bouchard, Ekeland and Touzi [4]. The asymptotic properties of the resulting numerical algorithm for the computation of the price of American put options (and more generally, for the expected value of functions of the solutions of reflected backward stochastic differential equations) have been analyzed by Bouchard and Touzi [5]. The main idea is to use the Malliavin integration by parts formula in order to get rid of the Dirac point masses in (62). In doing so one gets:

$$
\mathbb{E}\{A \mid B=b\}=\frac{\mathbb{E}\left\{A H_{b}(B) S\right\}}{\mathbb{E}\left\{H_{b}(B) S\right\}}
$$

where $H_{b}(x)=\prod_{i=1}^{p} \mathbf{1}_{\left[b_{i}, \infty\right)}\left(x_{i}\right)$, and $S$ is some non-negative random variable. An important consequence of this formula is the fact that the associated Monte Carlo estimator:

$$
\hat{E}[A \mid B=b]=\frac{\frac{1}{N} \sum_{s=1}^{N} A^{(s)} H_{b}\left(B^{(s)}\right) S^{(s)}}{\frac{1}{N} \sum_{s=1}^{N} H_{b}\left(B^{(s)}\right) S^{(s)}},
$$

constructed from an independent sample $\left\{\left(A^{(s)}, B^{(s)}, S^{(s)}\right)\right\}_{s=1, \cdots, N}$ of size $N$, converges at the $\sqrt{N}$-rate by the classical central limit theorem. The following subsection is devoted to a selfcontained derivation of these facts. We use a pedestrian approach based on the log-normality of our Gaussian framework, without ever appealing to results of the Malliavin calculus.

6.3. Integration-by-Parts based Regression Estimation. We first concentrate on a regression function of the form:

$$
r_{h}(x):=\mathbb{E}\left\{g\left(W_{t+h}\right) \mid W_{t}=x\right\}=\frac{\mathbb{E}\left\{g\left(W_{t+h}\right) \delta_{x}\left(W_{t}\right)\right\}}{\mathbb{E}\left\{\delta_{x}\left(W_{t}\right)\right\}}
$$

Integration by Parts. Let us denote by $\varphi$ the density of the standard one dimensional normal distribution, and let us assume that $g$ is a smooth function with a bounded derivative. By the independence of the increments of the Brownian motion, we have:

$$
\mathbb{E}\left\{g\left(W_{t+h}\right) \delta_{x}\left(W_{t}\right)\right\}=\iint g\left(w_{1}+w_{2}\right) \delta_{x}\left(w_{1}\right) \varphi\left(\frac{w_{1}}{\sqrt{t}}\right) \varphi\left(\frac{w_{2}}{\sqrt{h}}\right) d w_{1} d w_{2} .
$$

Integrating by parts with respect to the $w_{1}$ variable, we get :

$$
\begin{aligned}
\mathbb{E}\left\{g\left(W_{t+h}\right) \delta_{x}\left(W_{t}\right)\right\}= & \iint g\left(w_{1}+w_{2}\right) \mathbf{1}_{[x, \infty)}\left(w_{1}\right) \frac{w_{1}}{t} \varphi\left(\frac{w_{1}}{\sqrt{t}}\right) \varphi\left(\frac{w_{2}}{\sqrt{h}}\right) d w_{1} d w_{2} \\
& -\iint g^{\prime}\left(w_{1}+w_{2}\right) \mathbf{1}_{[x, \infty)}\left(w_{1}\right) \varphi\left(\frac{w_{1}}{\sqrt{t}}\right) \varphi\left(\frac{w_{2}}{\sqrt{h}}\right) d w_{1} d w_{2} .
\end{aligned}
$$


Next, we compute the second integral by integrating by parts with respect to the $w_{2}$ variable. We get:

$$
\begin{aligned}
\mathbb{E}\left\{g\left(W_{t+h}\right) \delta_{x}\left(W_{t}\right)\right\}= & \iint g\left(w_{1}+w_{2}\right) \mathbf{1}_{[x, \infty)}\left(w_{1}\right) \frac{w_{1}}{t} \varphi\left(\frac{w_{1}}{\sqrt{t}}\right) \varphi\left(\frac{w_{2}}{\sqrt{h}}\right) d w_{1} d w_{2} \\
& -\iint g\left(w_{1}+w_{2}\right) \mathbf{1}_{[x, \infty)}\left(w_{1}\right) \frac{w_{2}}{h} \varphi\left(\frac{w_{1}}{\sqrt{t}}\right) \varphi\left(\frac{w_{2}}{\sqrt{h}}\right) d w_{1} d w_{2} \\
= & \mathbb{E}\left\{g\left(W_{t+h}\right) \mathbf{1}_{[x, \infty)}\left(W_{t}\right) S_{h}\right\}
\end{aligned}
$$

where the random variable

$$
S_{h}=\frac{W_{t}}{t}-\frac{W_{t+h}-W_{t}}{h}
$$

is independent of the function $g$. Notice that formula (67) is established for a function $g \in C_{b}^{1}$. However, since it does not involve the regularity of $g$, we can conclude by a classical density argument that it is valid whenever $g\left(W_{t+h}\right) \in L^{2}$.

Actual Simulation. Let $\left(W^{(s)}\right)_{1 \leq s \leq S}$ be $n$ independent samples of the Wiener process $W$. Then, the Monte Carlo estimator suggested by the above formula is defined by

$$
\hat{r}_{N}(x):=\frac{\hat{q}_{N}[g](x)}{\hat{q}_{N}[1](x)} \quad \text { where } \quad \hat{q}_{N}[g](x):=\frac{1}{N} \sum_{s=1}^{N} g\left(W_{t+h}^{(s)}\right) \mathbf{1}_{[x, \infty)}\left(W_{t}^{(s)}\right) S_{h}^{(s)}
$$

where $S_{h}^{(s)}$ is computed from the sample $W^{(s)}$ using formula (68). Its asymptotic properties are directly deduced from the law of large numbers and the central limit theorem for independent identically distributed random variables. In particular, the rate of convergence is of the order $\sqrt{N}$.

The price to pay in order to recover the $\sqrt{N}$ rate of convergence is that the variance of the estimator $\hat{q}_{N}[g](x)$ explodes as $h$ shrinks to zero since

$$
\limsup _{h \searrow 0} S_{h}=\infty \quad \text { and } \quad \liminf _{h \searrow 0} S_{h}=-\infty
$$

Since our objective is to send the time step $h$ to zero, it is necessary to find a remedy to this variance explosion problem. 
Localization. In order to do so, we introduce a localization function. Let $\chi$ be an arbitrary smooth function with $\chi(0)=1$. Following the computations leading to formula (67) we get:

$$
\begin{aligned}
\mathbb{E}\left\{g\left(W_{t+h}\right) \delta_{x}\left(W_{t}\right)\right\}= & \mathbb{E}\left\{g\left(W_{t+h}\right) \delta_{x}\left(W_{t}\right) \chi\left(W_{t}-x\right)\right\} \\
= & \iint g\left(w_{1}+w_{2}\right) \delta_{x}\left(w_{1}\right) \chi\left(w_{1}-x\right) \varphi\left(\frac{w_{1}}{\sqrt{t}}\right) \varphi\left(\frac{w_{2}}{\sqrt{h}}\right) d w_{1} d w_{2} \\
= & \iint g\left(w_{1}+w_{2}\right) \mathbf{1}_{[x, \infty)}\left(w_{1}\right) \chi\left(w_{1}-x\right) \frac{w_{1}}{t} \varphi\left(\frac{w_{1}}{\sqrt{t}}\right) \varphi\left(\frac{w_{2}}{\sqrt{h}}\right) d w_{1} d w_{2} \\
& -\iint g^{\prime}\left(w_{1}+w_{2}\right) \mathbf{1}_{[x, \infty)}\left(w_{1}\right) \chi\left(w_{1}-x\right) \varphi\left(\frac{w_{1}}{\sqrt{t}}\right) \varphi\left(\frac{w_{2}}{\sqrt{h}}\right) d w_{1} d w_{2} \\
& -\iint g\left(w_{1}+w_{2}\right) \mathbf{1}_{[x, \infty)}\left(w_{1}\right) \chi^{\prime}\left(w_{1}-x\right) \varphi\left(\frac{w_{1}}{\sqrt{t}}\right) \varphi\left(\frac{w_{2}}{\sqrt{h}}\right) d w_{1} d w_{2} \\
= & \iint g\left(w_{1}+w_{2}\right) \mathbf{1}_{[x, \infty)}\left(w_{1}\right) \chi\left(w_{1}-x\right) \frac{w_{1}}{t} \varphi\left(\frac{w_{1}}{\sqrt{t}}\right) \varphi\left(\frac{w_{2}}{\sqrt{h}}\right) d w_{1} d w_{2} \\
& -\iint g\left(w_{1}+w_{2}\right) \mathbf{1}_{[x, \infty)}\left(w_{1}\right) \chi\left(w_{1}-x\right) \frac{W_{2}}{h} \varphi\left(\frac{w_{1}}{\sqrt{t}}\right) \varphi\left(\frac{w_{2}}{\sqrt{h}}\right) d w_{1} d w_{2} \\
& -\iint g\left(w_{1}+w_{2}\right) \mathbf{1}_{[x, \infty)}\left(w_{1}\right) \chi^{\prime}\left(w_{1}-x\right) \varphi\left(\frac{w_{1}}{\sqrt{t}}\right) \varphi\left(\frac{w_{2}}{\sqrt{h}}\right) d w_{1} d w_{2} \\
= & \mathbb{E}\left\{g\left(W_{t+h}\right) \mathbf{1}_{[x, \infty)}\left(W_{t}\right) S_{h, \chi}\right\},
\end{aligned}
$$

where the random variable $S_{h, \chi}$ is defined by:

$$
\begin{aligned}
S_{h, \chi} & =\chi\left(W_{t}-x\right)\left(\frac{W_{t}}{t}-\frac{W_{t+h}-W_{t}}{h}\right)-\chi^{\prime}\left(W_{t}-x\right) \\
& =\chi\left(W_{t}-x\right) S_{h}-\chi^{\prime}\left(W_{t}-x\right)
\end{aligned}
$$

is again independent of the function $g$. For each localization function $\chi$, one can now define a new Monte Carlo estimator as before. All these estimators share the nice convergence property at the $\sqrt{N}$-rate. Therefore, the natural question is whether one can reduce the variance of the Monte Carlo estimator by some convenient choice of localization function $\chi$.

4. Variance Reduction by Localization. Set $G:=g\left(W_{t+h}\right)^{2}$, and let us consider the integrated mean square error

$$
J(\chi):=\int_{\mathbb{R}} \mathbb{E}\left\{G \mathbf{1}_{W_{t}>x} S_{h, \chi}^{2}\right\} d x
$$

We are interested in the integrated mean square error minimization :

$$
V:=\min \{J(\chi): \chi \text { smooth, bounded and } \chi(0)=1\}
$$


Using Fubini's theorem and a path by path substitution, we get:

$$
\begin{aligned}
J(\chi) & =\mathbb{E}\left\{G \int_{-\infty}^{W_{t}}\left|\chi\left(W_{t}-x\right) S_{h}-\chi^{\prime}\left(W_{t}-x\right)\right|^{2} d x\right\} \\
& =\mathbb{E}\left\{G \int_{0}^{+\infty}\left|\chi(y) S_{h}-\chi^{\prime}(y)\right|^{2} d y\right\} .
\end{aligned}
$$

Observing that $\mathbb{E}\left\{G S_{h}\right\}=0$, this provides

$$
J(\chi)=\int_{0}^{+\infty}\left[\mathbb{E}\left\{G S_{h}^{2}\right\}|\chi(y)|^{2}+\mathbb{E}\{G\}\left|\chi^{\prime}(y)\right|^{2}\right] d y .
$$

Hence the integrated mean square error minimization is reduced to a classical problem of calculus of variations, which can be solved explicitly. The optimal localization function is then given by

$$
\chi_{h}(x):=e^{-\eta_{h} x} \quad \text { where } \quad \eta_{h}:=\left(\frac{\mathbb{E}\left\{G S_{h}^{2}\right\}}{\mathbb{E}\{G\}}\right)^{1 / 2} .
$$

In particular, this shows that

$$
\eta_{h}=\mathrm{O}\left(h^{-1 / 2}\right)
$$

6.4. Monte Carlo Estimation for the Finite Maturity Problem. We now return to the problem of the optimal multiple stopping problem, and more precisely to the pricing of swing options in the framework of the discrete time approximation set up in Subsection 6.1. Let $N_{n}$ be some integer depending on the time step parameter $n$, and let $\left\{W^{(s)}, 1 \leq s \leq N_{n}\right\}$ be $N_{n}$ independent samples of the Wiener process. For each integer $s$, we denote by $X^{(s)}$ the process $X$ associated to the Brownian motion $W^{(s)}$ via formula (16). Also, we set:

$$
\begin{aligned}
R_{h}^{(s)}\left(t_{j}, x\right) & :=S_{h, \chi_{h}}^{(s)}\left(t_{j}\right) \mathbf{1}_{[x, \infty)}\left(W_{t_{j}}^{(s)}\right) \\
& =\chi_{h}\left(W_{t}^{(s)}-x\right)\left[\eta_{h}+h^{-1}\left(2 W_{t_{j}}^{(s)}-W_{t_{j}-h}^{(s)}-W_{t_{j}+h}^{(s)}\right)\right] \mathbf{1}_{[x, \infty)}\left(W_{t_{j}}^{(s)}\right),
\end{aligned}
$$

where $S_{h}$ and $\chi_{h}$ are defined respectively in (69) and (71). Following the discussion of the previous paragraph, we define the estimators :

$$
\begin{aligned}
\tilde{\rho}_{n}^{(i)}\left(t_{j}, X_{t_{j}}\right):= & \frac{\frac{1}{N_{n}} \sum_{s=1}^{S} \hat{v}_{n}^{(i)}\left(t_{j+1}, X_{t_{j+1}}^{(s)}\right) R_{1 / n}^{(s)}\left(t_{j}, X_{t_{j}}\right)}{\frac{1}{N_{n}} \sum_{s=1}^{S} R_{1 / n}^{(s)}\left(t_{j}, W_{t_{j}}\right)}, \\
\tilde{\phi}_{n}^{(i)}\left(t_{j}, X_{t_{j}}\right):= & \phi\left(X_{t_{j}}\right) \\
& +e^{-r \delta} \frac{\frac{1}{N_{n}} \sum_{s=1}^{S} \hat{v}_{n}^{(i-1)}\left(t_{j}+\delta, X_{t_{j}+\delta}^{(s)}\right) R_{\delta}^{(s)}\left(t_{j}, X_{t_{j}}\right)}{\frac{1}{N_{n}} \sum_{s=1}^{S} R_{\delta}^{(s)}\left(t_{j}, W_{t_{j}}\right)} \mathbf{1}_{t_{j} \leq 1-\delta}
\end{aligned}
$$

of $\rho_{n}^{(i)}\left(t_{j}, X_{t_{j}}\right)$ and $\phi_{n}^{(i)}\left(t_{j}, X_{t_{j}}\right)$ respectively. These estimators are defined inductively, given the estimators $\hat{v}^{(i-1)}(.,$.$) and the previous estimator \hat{v}_{n}^{(i)}\left(t_{j+1}, X_{t_{j+1}}^{(s)}\right)$ in the backward procedure. Finally, 
we observe that $\rho_{n}^{(i)} \leq i K$ and $\phi_{n}^{(i)} \leq i K$. Hence, in order to ovoid an explosion of the algorithm, we define the truncated estimators (see [5]) :

$$
\begin{aligned}
& \hat{\rho}_{n}^{(i)}\left(t_{j}, X_{t_{j}}\right):=(i K) \wedge \tilde{\rho}_{n}^{(i)}\left(t_{j}, X_{t_{j}}\right)^{+}, \\
& \hat{\phi}_{n}^{(i)}\left(t_{j}, X_{t_{j}}\right):=(i K) \wedge \tilde{\phi}_{n}^{(i)}\left(t_{j}, X_{t_{j}}\right)^{+},
\end{aligned}
$$

and

$$
\hat{v}_{n}^{(i)}\left(t_{j}, X_{t_{j}}\right):=\max \left\{\hat{\rho}_{n}^{(i)}\left(t_{j}, X_{t_{j}}\right), \hat{\phi}_{n}^{(i)}\left(t_{j}, X_{t_{j}}\right)\right\}
$$

According to the error estimate of [5], in order for the approximation error to be of the order of $n^{-1 / 2}$, one has to choose a number $N_{n}$ of simulated trajectories such that

$$
N_{n}=\mathrm{O}\left(n^{7 / 2}\right) \text {. }
$$

The Value Functions. The above algorithm was implemented and tested for the swing put option with the following characteristics: maturity $T=1$ year, refraction period $\delta=0.1, r=.05, \sigma=.30$, maximal number of exercise rights $\ell=5, n=50$.

Monte Carlo Swing Value Functions for One Exercise Right

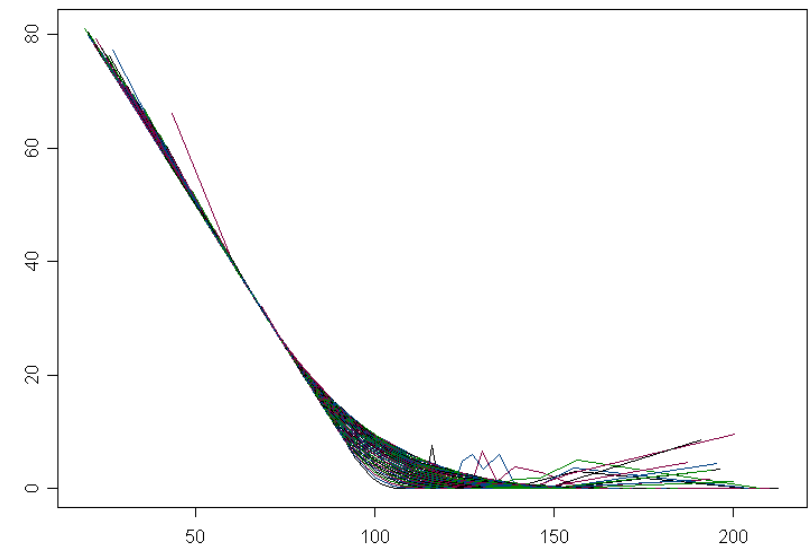

Monte Carlo Swing Value Functions for Three Exercise Rights

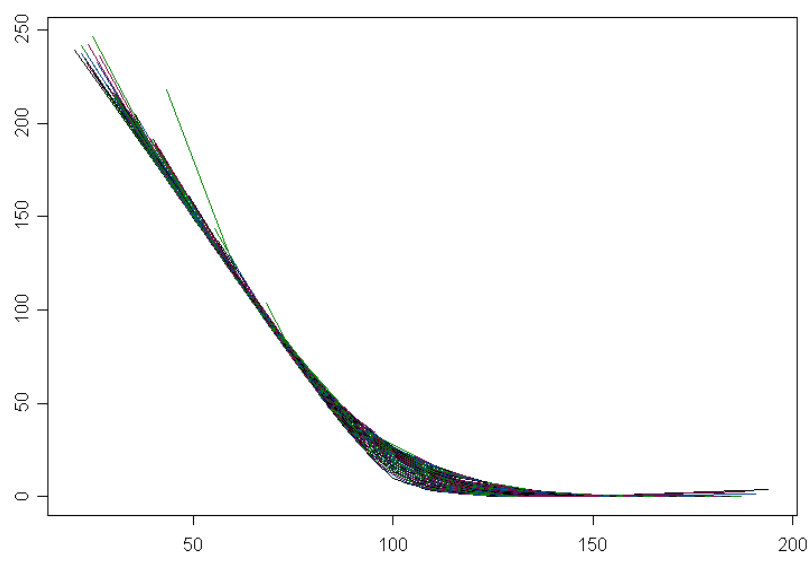

FIGURE 6. Graphs of the functions $v^{(1)}(t, \cdot)$ for $t=.59, .58, \cdots, .02, .01$ (left) and of the functions $v^{(3)}(t, \cdot)$ for $t=.49, .47, \cdots, .02, .01$ (right).

The left pane of Figure 6 gives the plots of the graphs of the functions $x \hookrightarrow v^{(1)}(t, x)$ for $t=$ $.59, .58, \cdots, .02, .01$. Two remarks are in order. First, these graphs are not computed over the same range of values of $x$. Essentially, we computed the values of $v^{(k)}(t, x)$ for the values of $x$ which can be reached by the sample paths of the diffusion process $X_{t}$, and we determined this range of values of $x$ from the results of our simulations. The second remark concerns the noise in the numerical results. Obviously, we should expect zero in the right hand side of the plots, and we see quite significant departures from this expectation. The right pane of the figure gives the plots of the graphs of the functions $x \hookrightarrow v^{(3)}(t, x)$ for $t=.59, .58, \cdots, .02, .01$. for $t=.49, .47, \cdots, .02, .01$. 


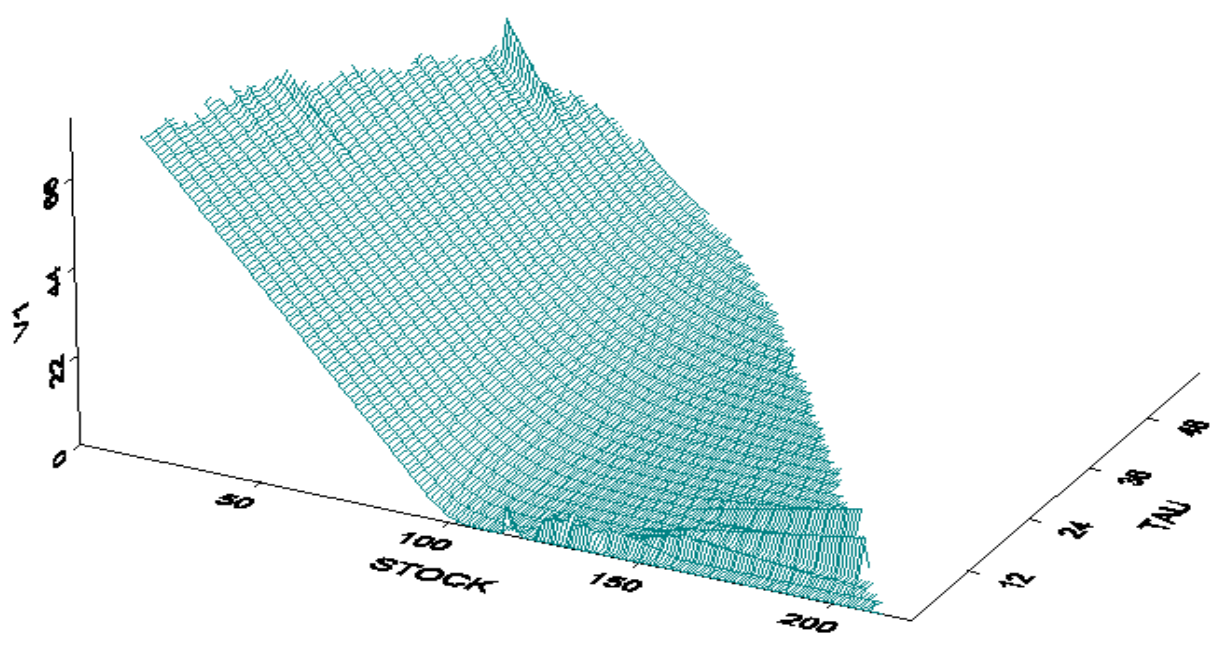

FIGURE 7. Surface plot of the graph of the function $v^{(1)}$ when regarded as function of both $t$ and $x$. The variable TAU represents $100 *(T-t)$.

Figure 7 gives the same plot as the left pane of Figure 6, but instead of super-imposing the onedimensional graphs on the same plot, we use both the $t$ and the $x$ variables to produce surface plots, or to be more specific the scaled time to maturity $\tau=100(T-t)$ and $x$. The fact that the range of $x$ varies with $t$ is obvious from this surface plot, and as expected, it is limited by some form of parabola. Plotting the graphs of the other value functions $v^{(k)}$ would produce very similar results and we refrain from producing them.

Number of Monte Carlo Scenarios. We present some partial numerical results to illustrate the effect of the number of trajectories $N_{n}$. According to the result of [5] which we re-derived above, the number $N_{n}$ should be of the order of $n^{7 / 2}$. The results collected in the following Table 1 show that a very high precision can be achieved even with a significantly smaller number of simulated trajectories.

Table 1. Swing put option values for various numbers of simulations $T=1$ year, $\delta=0.1$ year, $S_{0}=K=100, r=.05, \sigma=.30, n=50$ 


\begin{tabular}{|c|c|c|}
\hline \hline & $N=8,192$ & $N=16,384$ \\
\hline$v^{(1)}$ [stand. dev.] & $9.84[.22 \%]$ & $9.85[.12 \%]$ \\
$v^{(2)}$ [stand. dev.] & $19.21[.56 \%]$ & $19.26[.30 \%]$ \\
$v^{(3)}$ [stand. dev.] & $28.69[.68 \%]$ & $28.80[.30 \%]$ \\
$v^{(4)}$ [stand. dev.] & $38.34[.57 \%]$ & $38.48[.27 \%]$ \\
$v^{(5)}$ [stand. dev.] & $48.17[.50 \%]$ & $48.32[.30 \%]$ \\
\hline \hline
\end{tabular}

Exercise Regions. Next we identify an estimate of the exercise region for each of the value functions $v^{(i)}$ considered as a single stopping problem associated to the reward function $\phi^{(i)}(t, x)$. The corresponding exercise boundaries are given by the graphs of the functions $t \hookrightarrow \hat{x}_{i}^{*}(t)$. Estimates of these boundaries computed with the Monte Carlo method described in this section are plotted in Figure 8 . The computations were performed with the following parameters: maturity $T=10$ months, refraction period $\delta=2$ months, $r=.05, \sigma=.30$, maximal number of exercise rights $\ell=5, n=50$, $N=8192$. As expected these exercise boundaries are increasing functions of the time-to-maturity variable. We also verify that $\hat{x}_{i}^{*}(t) \geq \hat{x}_{i-1}^{*}(t)$. This property is consistent with the intuition. We proved rigorously this result in the case of the perpetual put options in Lemma 5, but a proof of this fact in the finite maturity case is still lacking: this monotonicity remains an interesting open problem.

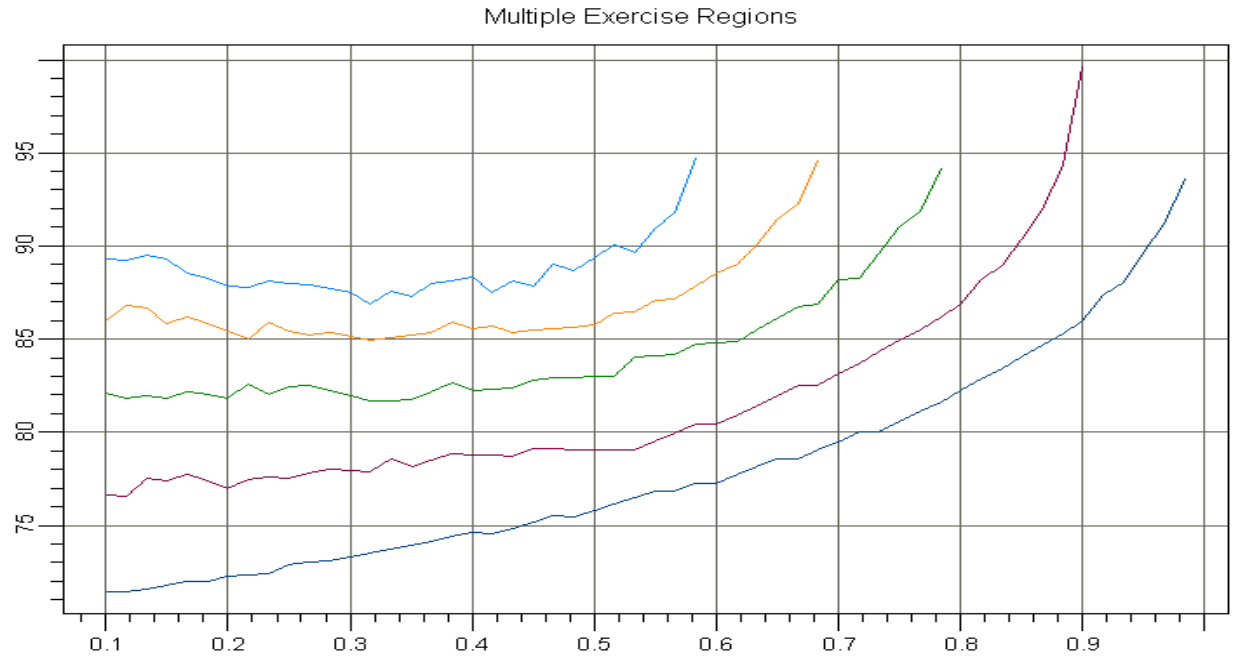

FIGURE 8. Estimates of the boundaries of the exercise regions of swing options with $\ell=5$ exercise rights, as given by the graphs of the functions $t \hookrightarrow x_{i}^{*}(t)$ computed via the Monte Carlo procedure described in the text. 


\section{REFERENCES}

[1] V. Bally and C. Pages (2003): A quantization algorithm for solving multidimensional discrete time optimal stopping problems. Bernoulli 9 (6) 1003 - 1049.

[2] A. Barbieri and M.B. Garman (2002): Understanding the Valuation of Swing Contracts. FEA Tech. Rep.

[3] D. Bosq (1998): Non-parametric Statistics for Stochastic Processes, Springer Verlag, New York.

[4] B. Bouchard, I. Ekeland, and N. Touzi (2002): On the Malliavin approach to Monte Carlo approximation of conditional expectations, Finance and Stochastics (to appear).

[5] B. Bouchard and N. Touzi (2002): Discrete Time Approximation and Monte-Carlo Simulation of Backward Stochastic Differential Equations, preprint.

[6] R. Cairoli and R. Dalang (1996): Sequential stochastic optimization. Wiley.

[7] R. Carmona and S. Dayanik (2003): Optimal Multiple Stopping of Linear Diffusions and Swing Options. Princeton University (tech. rep)

[8] J. F. Carrière (1996): Valuation of the Early-Exercise Price for Options using Simulations and Nonparametric Regression, Insurance : mathematics and Economics, 19, 19-30.

[9] E. Clément, D. Lamberton, and P. Protter (2002). An analysis of a least squares regression method for American option pricing, Finance and Stochastics, 6, 449-472.

[10] L. Clewlow, C. Strickland and V. Kaminski (2002): Risk analysis of swing contracts. EPRM, ??, ??-??.

[11] D. Egloff and M. Min-Oo (2002). Conergence of Monte Carlo algorithms for pricing American options, preprint.

[12] N. El Karoui (1981): Les aspects probabilistes du controle stochastique, Lect. Notes in Math \# 876, 73-238. Springer Verlag, New York N.Y.

[13] E. Fournié, J.M. Lasry, J. Lebuchoux, and P.L. Lions (2001): Applications of Malliavin calculus to Monte Carlo methods in finance II, Finance and Stochastics, 5, 201-236.

[14] G. Haggstrom (1967): Optimal sequential procedures when more than one stop is required. Ann. Math. Stat. 381618 - 1626

[15] A. Ibanez (2002): Valuation by Simulation of Contingent Claims with Multiple Early Exercises. (preprint)

[16] P. Jaillet, E.I Ronn and S. Tompaidis (2002): Valuation of Commodity-Based Swing Options. Univ. Texas, Austin (preprint).

[17] I. Karatzas and S. Shreve (2000): Mathematical Finance. Springer Verlag New York, NY.

[18] D. Lamberton and B. Lapeyre (1996): Introduction to Stochastic Calculus Applied to Finance. Chapman \& Hall New York, NY.

[19] P.L. Lions and H. Regnier (2001): Calcul du prix et des sensibilités d'une option américaine par une méthode de Monte Carlo, preprint.

[20] F. A. Longstaff and R. S. Schwartz (2001). Valuing American Options By Simulation : A simple Least-Square Approach, Review of Financial Studies, 14, 113-147.

[21] C. Martini and C. Patry (1999): Variance Optimal Hedging in the Black-Scholes Model for a given Number of Transactions. INRIA, Tech. Rep. 3767.

[22] N. Meinshausen and B.M. Hambly (2004): Monte Carlo methods for the valuation of multiple exercise options. Math. Finance

[23] J. Neveu (1972): Discrete Time Martingales. Masson, Paris.

[24] A.C. Thompson (1995): Valuation of Path-Dependent Contingent Claims with Multiple Exercise Decisions over Time: The Case of Take-or-Pay. Journal of Financial and Quantitative Analysis, 30, 271-293.

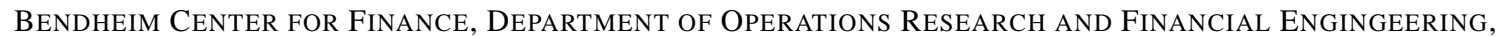
Princeton University, Princeton, NJ 08544

E-mail address: rcarmona@princeton.edu

CREST, 15 Bd Gabriel Peri, 92245 Malakoff Cedex, France

E-mail address: Nizar.Touzi@ensae.fr 